Supporting Information

\title{
LC-MS Identification, Isolation and Structural \\ Elucidation of Anti-HIV Tigliane Diterpenoids from Wikstroemia lamatsoensis
}

Mi Zhang, ${ }^{\dagger}$ Kouharu Otsuki, ${ }^{\dagger}$ Takashi Kikuchi, ${ }^{\dagger}$ Zi-Song Bai, ${ }^{\ddagger}$ Di Zhou, ${ }^{\ddagger}$ Li Huang, ${ }^{\S}$ Chin-Ho Chen, ${ }^{\S}$ Susan

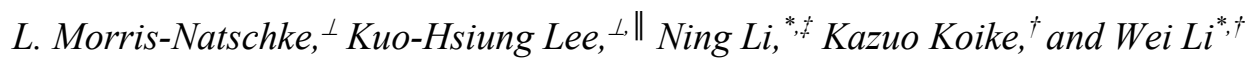

†Faculty of Pharmaceutical Sciences, Toho University, Miyama 2-2-1, Funabashi, Chiba 274-8510, Japan

†School of Traditional Chinese Materia Medica, Shenyang Pharmaceutical University, Shenyang 110016, People's Republic of China

${ }^{\S}$ Surgical Science, Department of Surgery, Duke University Medical Center, Durham, NC, 27710, United States

${ }^{\perp}$ Natural Products Research Laboratories, UNC Eshelman School of Pharmacy, University of North Carolina, Chapel Hill, NC, 27599, United States

$\|_{\text {Chinese Medicine Research and Development Center, China Medical University and Hospital, Taichung }}$ 404, Taiwan 


\section{Contents}

\section{Spectroscopic data}

Table S1. ESIMS data of compounds 1-8 in positive ion modes from W. lamatsoensis and MS/MS data obtained from the bold fragment ions.

Table S2. ESIMS data of compounds 1-8 in negative ion modes from W. lamatsoensis and MS/MS data

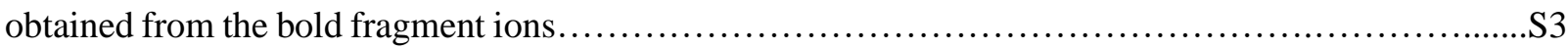

Figure S1. The proposed characteristic MS/MS fragmentation schemes of compounds 1-8..........S4

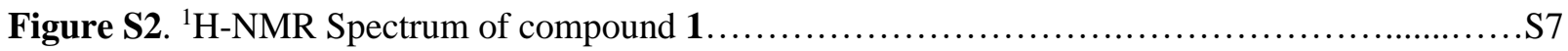

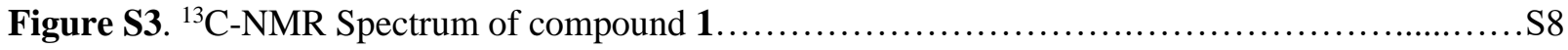

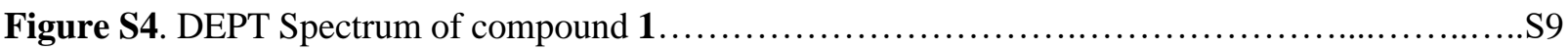

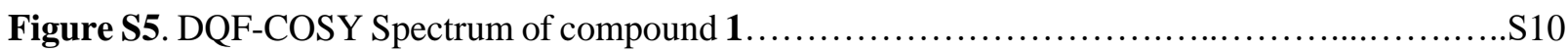

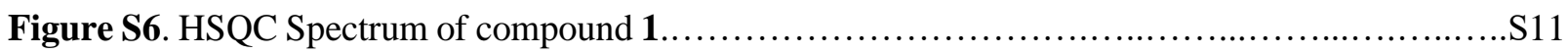

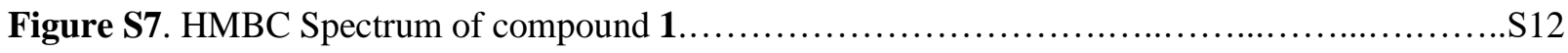

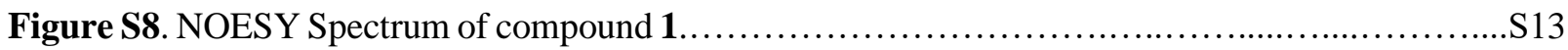

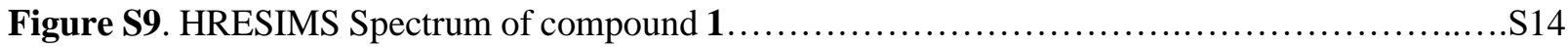

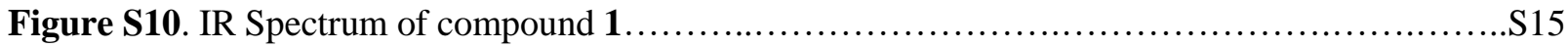

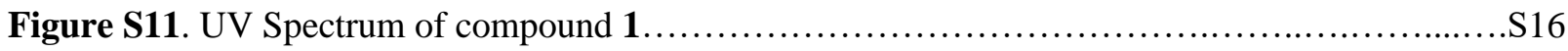

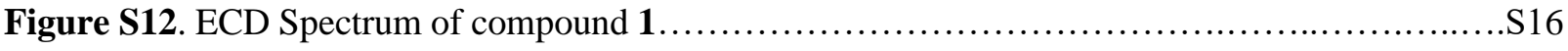

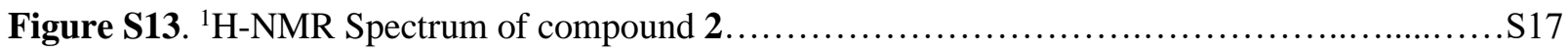

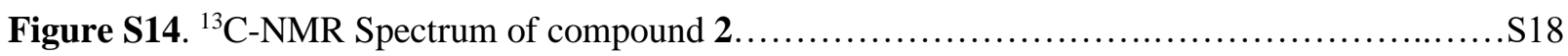

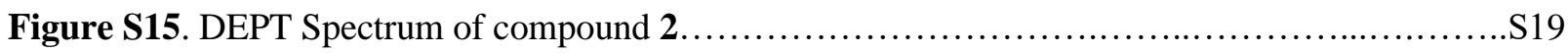

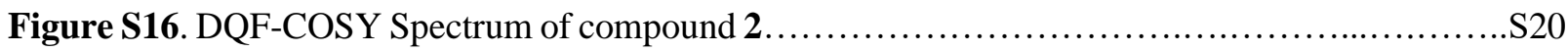

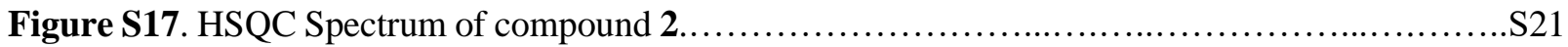

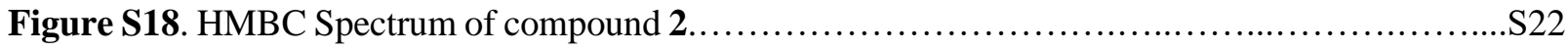

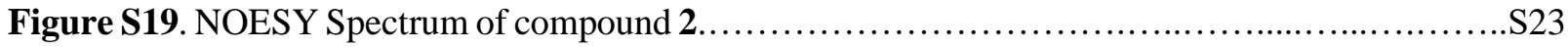

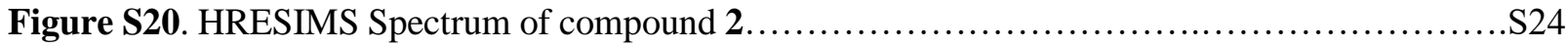

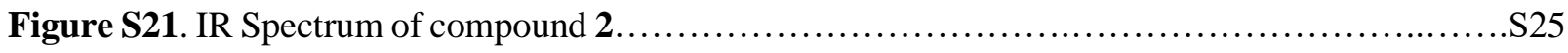

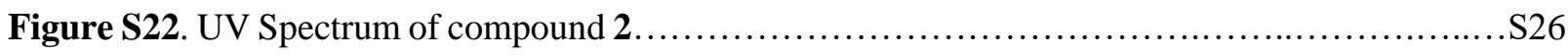

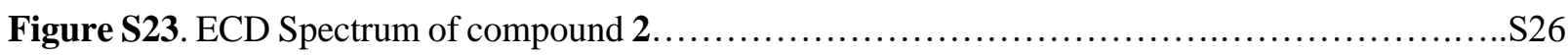


Table S1. ESIMS data of compounds 1-8 in positive ion modes from W. lamatsoensis and MS/MS data obtained from the bold fragment ions.

\begin{tabular}{|c|c|c|c|c|c|}
\hline No. & $\begin{array}{c}R_{\mathrm{t}} \\
(\min )\end{array}$ & $\begin{array}{l}\text { Molecular } \\
\text { formula }\end{array}$ & $\begin{array}{c}\text { MS on main ion } \\
\left(\mathrm{ESI}^{+}, m / z\right)(\text { relative intensity } \%)\end{array}$ & $\begin{array}{l}\text { HCD-MS/MS on main ion } \\
\quad \text { (relative intensity \%) }\end{array}$ & identification \\
\hline 1 & 3.93 & $\mathrm{C}_{28} \mathrm{H}_{40} \mathrm{O}_{7}$ & $\begin{array}{l}994(54)\left[2 \mathrm{M}+\mathrm{NH}_{4}\right]^{+}, \\
506(94)\left[\mathrm{M}+\mathrm{NH}_{4}\right]^{+}, \\
489(100)[\mathrm{M}+\mathrm{H}]^{+}, \\
471(19)\left[\mathrm{M}+\mathrm{H}-\mathrm{H}_{2} \mathrm{O}\right]^{+}, \\
345(17)\left[\mathrm{M}+\mathrm{H}-\mathrm{C}_{8} \mathrm{H}_{16} \mathrm{O}_{2}\right]^{+} \\
327(77)\left[\mathrm{M}+\mathrm{H}-\mathrm{C}_{8} \mathrm{H}_{16} \mathrm{O}_{2}-\mathrm{H}_{2} \mathrm{O}\right]^{+} \\
309(46)\left[\mathrm{M}+\mathrm{H}-\mathrm{C}_{8} \mathrm{H}_{16} \mathrm{O}_{2}-2 \mathrm{H}_{2} \mathrm{O}\right]^{+}\end{array}$ & $\begin{array}{l}327(19), 309 \text { (63), } 299(21), 291(12), 281(35), \\
279 \text { (10), } 241 \text { (100), } 239(16), 195(7), 133(18), \\
127(3)\end{array}$ & wikstrocin D \\
\hline 2 & 3.85 & $\mathrm{C}_{28} \mathrm{H}_{42} \mathrm{O}_{8}$ & $\begin{array}{l}1013(30)[2 \mathrm{M}+\mathrm{H}]^{+} \\
489(19)\left[\mathrm{M}+\mathrm{H}-\mathrm{H}_{2} \mathrm{O}\right]^{+} \\
471(13)\left[\mathrm{M}+\mathrm{H}-2 \mathrm{H}_{2} \mathrm{O}\right]^{+} \\
345(45)\left[\mathrm{M}+\mathrm{H}-\mathrm{C}_{8} \mathrm{H}_{16} \mathrm{O}_{2}-\mathrm{H}_{2} \mathrm{O}\right]^{+} \\
327(94)\left[\mathrm{M}+\mathrm{H}-\mathrm{C}_{8} \mathrm{H}_{16} \mathrm{O}_{2}-2 \mathrm{H}_{2} \mathrm{O}\right]^{+} \\
309(35)\left[\mathrm{M}+\mathrm{H}-\mathrm{C}_{8} \mathrm{H}_{16} \mathrm{O}_{2}-3 \mathrm{H}_{2} \mathrm{O}\right]^{+}\end{array}$ & $\begin{array}{l}345(52), 327(51), 309(59), 299(18), 293(25), \\
291(10), 281(26), 279(8), 269(54), 267(8), \\
263(11), 253(100), 251(10), 241(21), 239 \\
(14), 133(17), 127(17)\end{array}$ & wikstrocin $\mathrm{E}$ \\
\hline 3 & 4.34 & $\mathrm{C}_{35} \mathrm{H}_{46} \mathrm{O}_{9}$ & $\begin{array}{l}489(100)\left[\mathrm{M}+\mathrm{H}-\mathrm{C}_{7} \mathrm{H}_{6} \mathrm{O}_{2}\right]^{+} \\
471(11)\left[\mathrm{M}+\mathrm{H}-\mathrm{C}_{7} \mathrm{H}_{6} \mathrm{O}_{2}-\mathrm{H}_{2} \mathrm{O}\right]^{+}\end{array}$ & $\begin{array}{l}471(42), 453(54), 435(10), 407(8), 395(6), \\
373(56), 355(15), 345(58), 329(40), 327(92), \\
309(84), 299(10), 291(8), 281(19), 269(100), \\
251(6), 241(19), 239(6), 231(9), 229(11), \\
201(13), 179(26), 127(38)\end{array}$ & wikstrocin A \\
\hline 4 & 4.99 & $\mathrm{C}_{35} \mathrm{H}_{46} \mathrm{O}_{9}$ & $\begin{array}{l}471(100)\left[\mathrm{M}+\mathrm{H}-\mathrm{C}_{7} \mathrm{H}_{6} \mathrm{O}_{2}-\mathrm{H}_{2} \mathrm{O}\right]^{+}, \\
453(13)\left[\mathrm{M}+\mathrm{H}-\mathrm{C}_{7} \mathrm{H}_{6} \mathrm{O}_{2}-2 \mathrm{H}_{2} \mathrm{O}\right]^{+}, \\
327(5)\left[\mathrm{M}+\mathrm{H}-\mathrm{C}_{8} \mathrm{H}_{16} \mathrm{O}_{2}-\mathrm{C}_{7} \mathrm{H}_{6} \mathrm{O}_{2}-\mathrm{H}_{2} \mathrm{O}\right]^{+}\end{array}$ & $\begin{array}{l}453(24), 435(18), 345(24), 327(100), 309 \\
(70), \quad 297(53), 291(14), \quad 281(9), 279(7), \\
185(9), 127(12)\end{array}$ & wikstrocin B \\
\hline 5 & 6.27 & $\mathrm{C}_{35} \mathrm{H}_{44} \mathrm{O}_{9}$ & $\begin{array}{l}1239(6)[2 \mathrm{M}+\mathrm{Na}]^{+} \\
1234(6)\left[2 \mathrm{M}+\mathrm{NH}_{4}\right]^{+} \\
626(29)\left[\mathrm{M}+\mathrm{NH}_{4}\right]^{+} \\
\mathbf{4 8 7}(100)\left[\mathrm{M}+\mathrm{H}-\mathrm{C}_{7} \mathrm{H}_{6} \mathrm{O}_{2}\right]^{+}\end{array}$ & $\begin{array}{l}469(7), 361(11), 343(30), 325(6), 307(3), \\
297(6), 285(8), 267(8), 257(5), 239(6), 161 \\
(66), 151(16), 149(16), 133(7), 127(100), 109 \\
(6)\end{array}$ & dapholosericin A \\
\hline 6 & 5.86 & $\mathrm{C}_{35} \mathrm{H}_{46} \mathrm{O}_{10}$ & $\begin{array}{l}487(100)\left[\mathrm{M}+\mathrm{H}-\mathrm{C}_{7} \mathrm{H}_{6} \mathrm{O}_{2}-\mathrm{H}_{2} \mathrm{O}\right]^{+} \\
469(5)\left[\mathrm{M}+\mathrm{H}-\mathrm{C}_{7} \mathrm{H}_{6} \mathrm{O}_{2}-2 \mathrm{H}_{2} \mathrm{O}\right]^{+}\end{array}$ & $\begin{array}{l}469(58), 361(11), 343(30), 325(5), 307(3), \\
297(6), 285(8), 267(8), 257(5), 239(6), 161 \\
(66), 151(16), 149(16), 133(9), 127(100), 109 \\
(6)\end{array}$ & stelleracin E \\
\hline 7 & 5.36 & $\mathrm{C}_{35} \mathrm{H}_{46} \mathrm{O}_{9}$ & $\begin{array}{l}1221(8)[2 \mathrm{M}+\mathrm{H}]^{+}, \\
593(18)\left[\mathrm{M}+\mathrm{H}-\mathrm{H}_{2} \mathrm{O}\right]^{+}, \\
489(100)\left[\mathrm{M}+\mathrm{H}-\mathrm{C}_{7} \mathrm{H}_{6} \mathrm{O}_{2}\right]^{+} \\
471(35)\left[\mathrm{M}+\mathrm{H}-\mathrm{C}_{7} \mathrm{H}_{6} \mathrm{O}_{2}-\mathrm{H}_{2} \mathrm{O}\right]^{+}, \\
327(14)\left[\mathrm{M}+\mathrm{H}-\mathrm{C}_{8} \mathrm{H}_{16} \mathrm{O}_{2}-\mathrm{C}_{7} \mathrm{H}_{6} \mathrm{O}_{2}-\mathrm{H}_{2} \mathrm{O}\right]^{+} \\
309(9)\left[\mathrm{M}+\mathrm{H}-\mathrm{C}_{8} \mathrm{H}_{16} \mathrm{O}_{2}-\mathrm{C}_{7} \mathrm{H}_{6} \mathrm{O}_{2}-2 \mathrm{H}_{2} \mathrm{O}\right]^{+}\end{array}$ & $\begin{array}{l}453(6), 435(7), 345(11), 327(62), 309(100), \\
291(27), 283(35), 281(17), 267(6), 263(8), \\
145(5), 127(16)\end{array}$ & stelleracin C \\
\hline
\end{tabular}


$\mathrm{C}_{35} \mathrm{H}_{46} \mathrm{O}_{8} \quad 1189$ (20) $[2 \mathrm{M}+\mathrm{H}]^{+}$

$473(100)\left[\mathrm{M}+\mathrm{H}-\mathrm{C}_{7} \mathrm{H}_{6} \mathrm{O}_{2}\right]^{+}$

455 (14) $\left[\mathrm{M}+\mathrm{H}-\mathrm{C}_{7} \mathrm{H}_{6} \mathrm{O}_{2}-\mathrm{H}_{2} \mathrm{O}\right]^{+}$

455 (8), 437 (8), 329 (56), 311 (100), 293 (17), 283 (5), 267 (7), 127 (10)

2-O-benzoyl

$311(57)\left[\mathrm{M}+\mathrm{H}-\mathrm{C}_{8} \mathrm{H}_{16} \mathrm{O}_{2}-\mathrm{C}_{7} \mathrm{H}_{6} \mathrm{O}_{2}-\mathrm{H}_{2} \mathrm{O}\right.$ 
Table S2. ESIMS data of compounds 1-8 in negative ion modes from W. lamatsoensis and MS/MS data obtained from the [M+HCOO] $]^{-}$ions.

\begin{tabular}{|c|c|c|c|c|c|c|c|}
\hline \multirow[t]{2}{*}{ No. } & \multirow{2}{*}{$\begin{array}{c}R_{\mathrm{t}} \\
(\min )\end{array}$} & \multirow{2}{*}{$\begin{array}{l}\text { Molecular } \\
\text { formula }\end{array}$} & \multicolumn{2}{|c|}{$\left[\mathrm{M}+\mathrm{HCOO}^{-}(\mathrm{m} / \mathrm{z})\right.$} & \multirow{2}{*}{$\begin{array}{c}\text { Other fragment ions } \\
\left(\mathrm{ESI}^{-}, m / z\right)(\text { relative intensity } \%)\end{array}$} & \multirow{2}{*}{$\begin{array}{l}\text { HCD-MS/MS on main ion } \\
\quad \text { (relative intensity \%) }\end{array}$} & \multirow[t]{2}{*}{ identification } \\
\hline & & & $\begin{array}{c}\text { Detected } \\
\text { mass }(m / z)\end{array}$ & $\begin{array}{l}\text { Error } \\
(\mathrm{ppm})\end{array}$ & & & \\
\hline 1 & 3.93 & $\mathrm{C}_{28} \mathrm{H}_{40} \mathrm{O}_{7}$ & 533.2758 & 1.77 & $487(6)[\mathrm{M}-\mathrm{H}]^{-}$ & $\begin{array}{l}343(100), 325(99), 313(55), 295 \\
(14), 281(6), 263(7), 167(5), \\
143(89)\end{array}$ & wikstrocin D \\
\hline 2 & 3.85 & $\mathrm{C}_{28} \mathrm{H}_{42} \mathrm{O}_{8}$ & 551.2867 & 1.81 & $\begin{array}{l}487 \text { (33) }\left[\mathrm{M}-\mathrm{H}-\mathrm{H}_{2} \mathrm{O}\right]^{-}, \\
343(51)\left[\mathrm{M}-\mathrm{H}-\mathrm{C}_{8} \mathrm{H}_{16} \mathrm{O}_{2}-\mathrm{H}_{2} \mathrm{O}\right]^{-}, \\
325(9)\left[\mathrm{M}-\mathrm{H}-\mathrm{C}_{8} \mathrm{H}_{16} \mathrm{O}_{2}-2 \mathrm{H}_{2} \mathrm{O}\right]^{-}\end{array}$ & $\begin{array}{l}343(32), 325(19), 313(27), 295 \\
(5), 281(4), 263(2), 167(2), 163 \\
(5), 149(12), 144(9), 143(100)\end{array}$ & wikstrocin $\mathrm{E}$ \\
\hline 3 & 4.34 & $\mathrm{C}_{35} \mathrm{H}_{46} \mathrm{O}_{9}$ & 655.3122 & 1.29 & $-a$ & $\begin{array}{l}655(6), 591(6), 573(10), 447 \\
(6), 325(10), 307(62), 295(35), \\
195(36), 177(9), 175(5), 167 \\
(9), 143(33), 121(100)\end{array}$ & wikstrocin A \\
\hline 4 & 4.99 & $\mathrm{C}_{35} \mathrm{H}_{46} \mathrm{O}_{9}$ & 655.3128 & 2.31 & $-^{a}$ & $\begin{array}{l}417(6), 325(2), 295(3), 143 \\
(68), 121(100)\end{array}$ & wikstrocin B \\
\hline 5 & 6.27 & $\mathrm{C}_{35} \mathrm{H}_{44} \mathrm{O}_{9}$ & 653.2971 & 2.17 & $607(6)[\mathrm{M}-\mathrm{H}]^{-}$ & $\begin{array}{l}463(4), 433(4), 341(17), 323 \\
(85), 311(100), 305(8), 293(3), \\
143(21), 121(29)\end{array}$ & dapholosericin A \\
\hline 6 & 5.86 & $\mathrm{C}_{35} \mathrm{H}_{46} \mathrm{O}_{10}$ & 671.3074 & 1.65 & $\begin{array}{l}607(35)\left[\mathrm{M}-\mathrm{H}-\mathrm{H}_{2} \mathrm{O}\right]^{-} \\
341(6)\left[\mathrm{M}-\mathrm{H}-\mathrm{C}_{8} \mathrm{H}_{16} \mathrm{O}_{2}-\mathrm{C}_{7} \mathrm{H}_{6} \mathrm{O}_{2}-\mathrm{H}_{2} \mathrm{O}\right]^{-}\end{array}$ & $\begin{array}{l}341(5), 323(100), 311(62), 305 \\
(40), 293(8), 279(10), 143(54), \\
121(78)\end{array}$ & stelleracin E \\
\hline 7 & 5.36 & $\mathrm{C}_{35} \mathrm{H}_{46} \mathrm{O}_{9}$ & 655.3123 & 1.48 & $-^{a}$ & $\begin{array}{l}609(2), 579(5), 573(6), 343(2), \\
325(6), 307(7), 231(7), 143 \\
(70), 121(100)\end{array}$ & stelleracin $\mathrm{C}$ \\
\hline 8 & 6.21 & $\mathrm{C}_{35} \mathrm{H}_{46} \mathrm{O}_{8}$ & 639.3177 & 2.15 & $-^{a}$ & $\begin{array}{l}622(4), 593(41), 527(7), 471 \\
(14), 453(37), 309(3), 291(6) \\
143(100)\end{array}$ & $\begin{array}{l}\text { 12-O-benzoyl } \\
13 \text {-octanoate }\end{array}$ \\
\hline
\end{tabular}

$\overline{a_{-}, \text {not detected. }}$ 


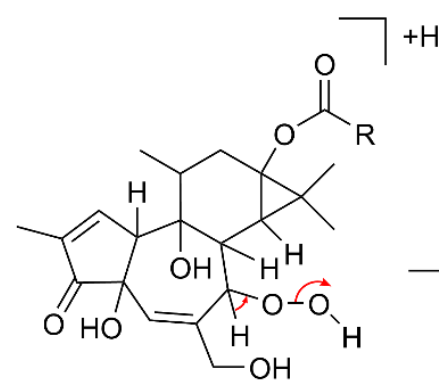

$\mathrm{C}_{28} \mathrm{H}_{43} \mathrm{O}_{8}(\mathrm{~m} / \mathrm{z} 507)$ $2 \mathrm{R}=\left(\mathrm{CH}_{2}\right)_{6} \mathrm{CH}_{3}$
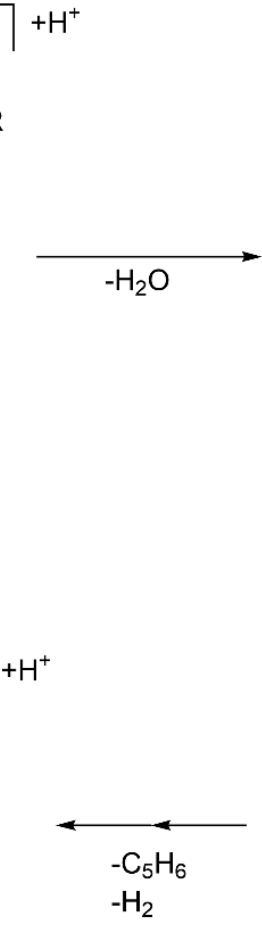

$\mathrm{C}_{15} \mathrm{H}_{13} \mathrm{O}_{3}(\mathrm{~m} / \mathrm{z} 241)$

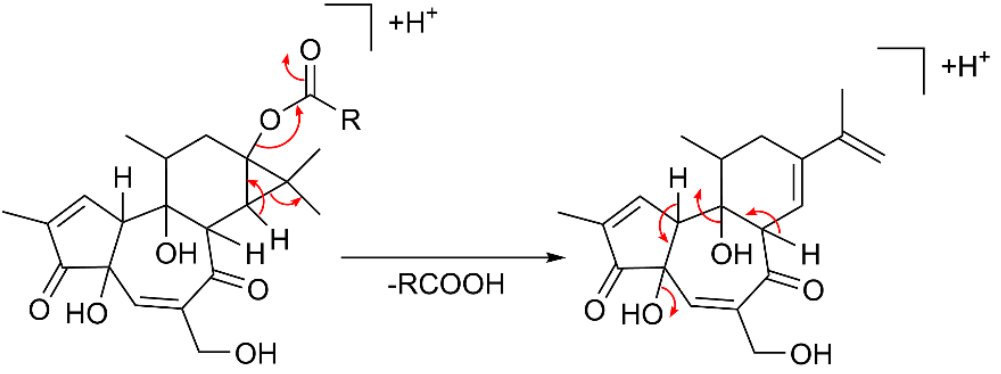

$\mathrm{C}_{28} \mathrm{H}_{41} \mathrm{O}_{7}(\mathrm{~m} / \mathrm{z} 489)$ $1 \mathrm{R}=\left(\mathrm{CH}_{2}\right)_{6} \mathrm{CH}_{3}$

$\mathrm{C}_{20} \mathrm{H}_{25} \mathrm{O}_{5}(m / z$ 345)

$-2 \mathrm{H}_{2} \mathrm{O}$

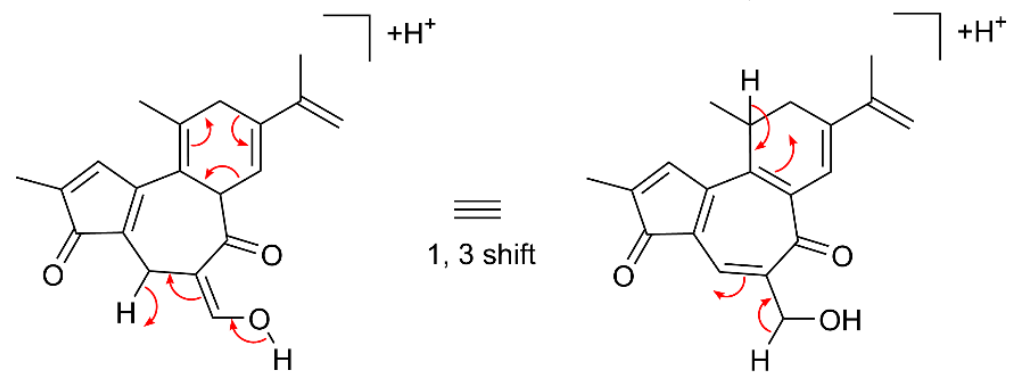

$\mathrm{C}_{20} \mathrm{H}_{21} \mathrm{O}_{3}(m / z$ 309)

$\mathrm{C}_{20} \mathrm{H}_{21} \mathrm{O}_{3}(\mathrm{~m} / \mathrm{z} 309)$

Figure S1. The proposed characteristic MS/MS fragmentation schemes of compounds 1-8. 


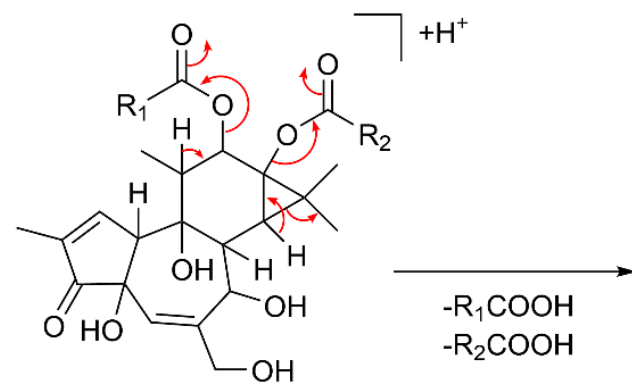

$\mathrm{C}_{35} \mathrm{H}_{47} \mathrm{O}_{9}(\mathrm{~m} / \mathrm{z} 611)$

$3 \mathrm{R}_{1}=\mathrm{Bz} \quad \mathrm{R}_{2}=\left(\mathrm{CH}_{2}\right)_{6} \mathrm{CH}_{3}$

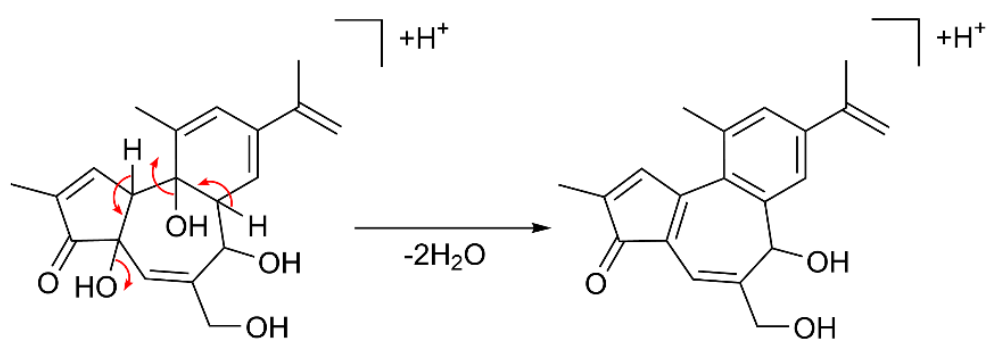

$\mathrm{C}_{20} \mathrm{H}_{25} \mathrm{O}_{5}(\mathrm{~m} / \mathrm{z} 345)$

$\mathrm{C}_{20} \mathrm{H}_{21} \mathrm{O}_{3}(\mathrm{~m} / \mathrm{z} 309)$

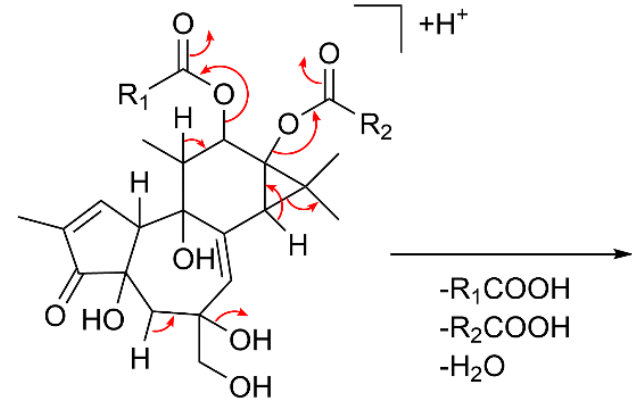

$\mathrm{C}_{35} \mathrm{H}_{47} \mathrm{O}_{9}(\mathrm{~m} / \mathrm{z} 611)$

$4 \mathrm{R}_{1}=\mathrm{Bz} \quad \mathrm{R}_{2}=\left(\mathrm{CH}_{2}\right)_{6} \mathrm{CH}_{3}$

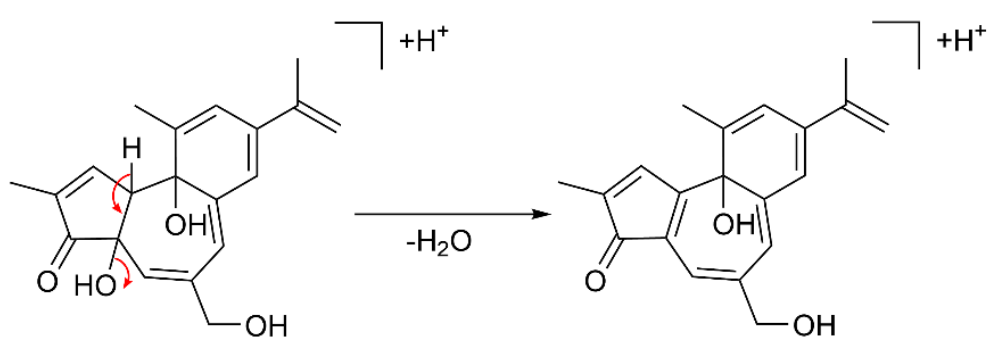

$\mathrm{C}_{20} \mathrm{H}_{23} \mathrm{O}_{4}(\mathrm{~m} / \mathrm{z} 327)$

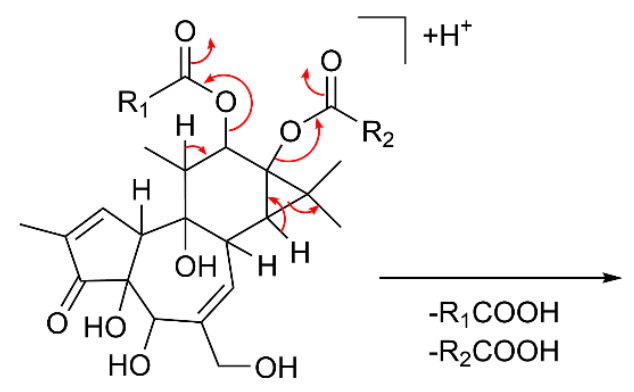

$\mathrm{C}_{35} \mathrm{H}_{47} \mathrm{O}_{9}(\mathrm{~m} / \mathrm{z} 611)$

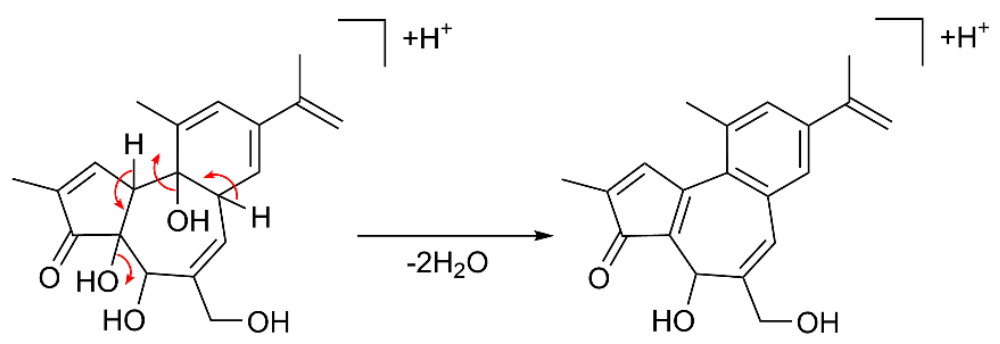

$\mathrm{C}_{20} \mathrm{H}_{25} \mathrm{O}_{5}(\mathrm{~m} / \mathrm{z} 345)$

$\mathrm{C}_{20} \mathrm{H}_{21} \mathrm{O}_{3}(\mathrm{~m} / \mathrm{z} 309)$

$7 \mathrm{R}_{1}=\mathrm{Bz} \quad \mathrm{R}_{2}=\left(\mathrm{CH}_{2}\right)_{6} \mathrm{CH}_{3}$

Figure S1. The proposed characteristic MS/MS fragmentation schemes of compounds 1-8 (continued). 


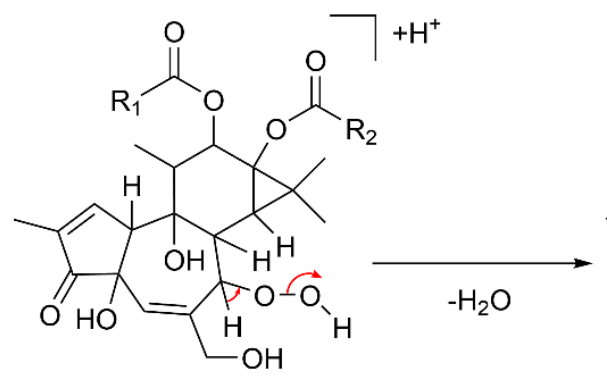

$\mathrm{C}_{35} \mathrm{H}_{47} \mathrm{O}_{10}(\mathrm{~m} / \mathrm{z} 627)$

$6 \mathrm{R}_{1}=\mathrm{Bz} \quad \mathrm{R}=\left(\mathrm{CH}_{2}\right)_{6} \mathrm{CH}_{3}$

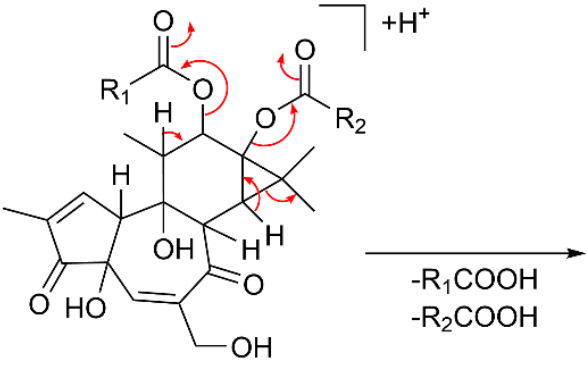

$\mathrm{C}_{35} \mathrm{H}_{45} \mathrm{O}_{9}(\mathrm{~m} / \mathrm{z} 609)$ $5 \mathrm{R}_{1}=\mathrm{Bz} \quad \mathrm{R}_{2}=\left(\mathrm{CH}_{2}\right)_{6} \mathrm{CH}_{3}$

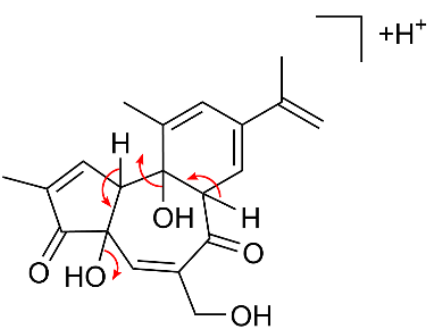

$\mathrm{C}_{20} \mathrm{H}_{25} \mathrm{O}_{5}(\mathrm{~m} / \mathrm{z} 343)$

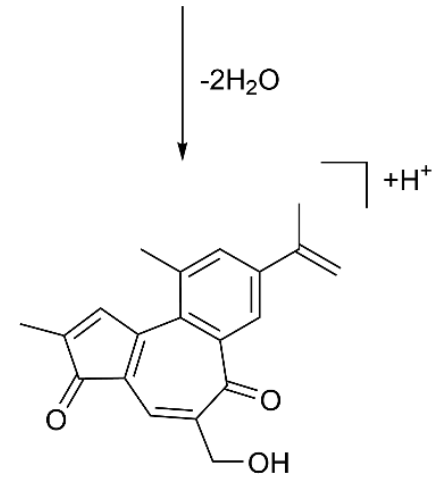

$\mathrm{C}_{20} \mathrm{H}_{21} \mathrm{O}_{3}(m / z 307)$

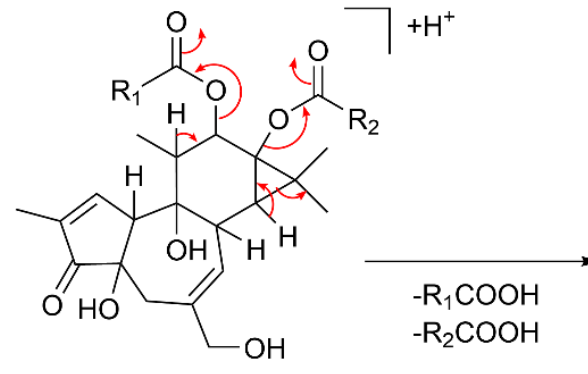

$\mathrm{C}_{35} \mathrm{H}_{47} \mathrm{O}_{8}(m / z 595)$

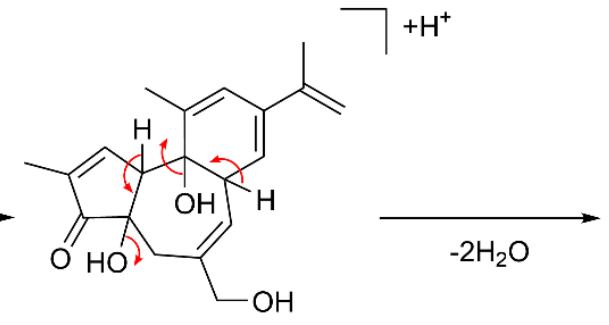

$\mathrm{C}_{20} \mathrm{H}_{25} \mathrm{O}_{4}(m / z 329)$<smiles>C=C(C)c1cc(C)c2c(c1)C=C(CO)CC1=C2C=C(C)C1=O</smiles>

$\mathrm{C}_{20} \mathrm{H}_{21} \mathrm{O}_{2}(m / z$ 293)

$8 \mathrm{R}_{1}=\mathrm{Bz} \mathrm{R} \mathrm{R}_{2}=\left(\mathrm{CH}_{2}\right)_{6} \mathrm{CH}_{3}$

Figure S1. The proposed characteristic MS/MS fragmentation schemes of compounds 1-8 (continued). 


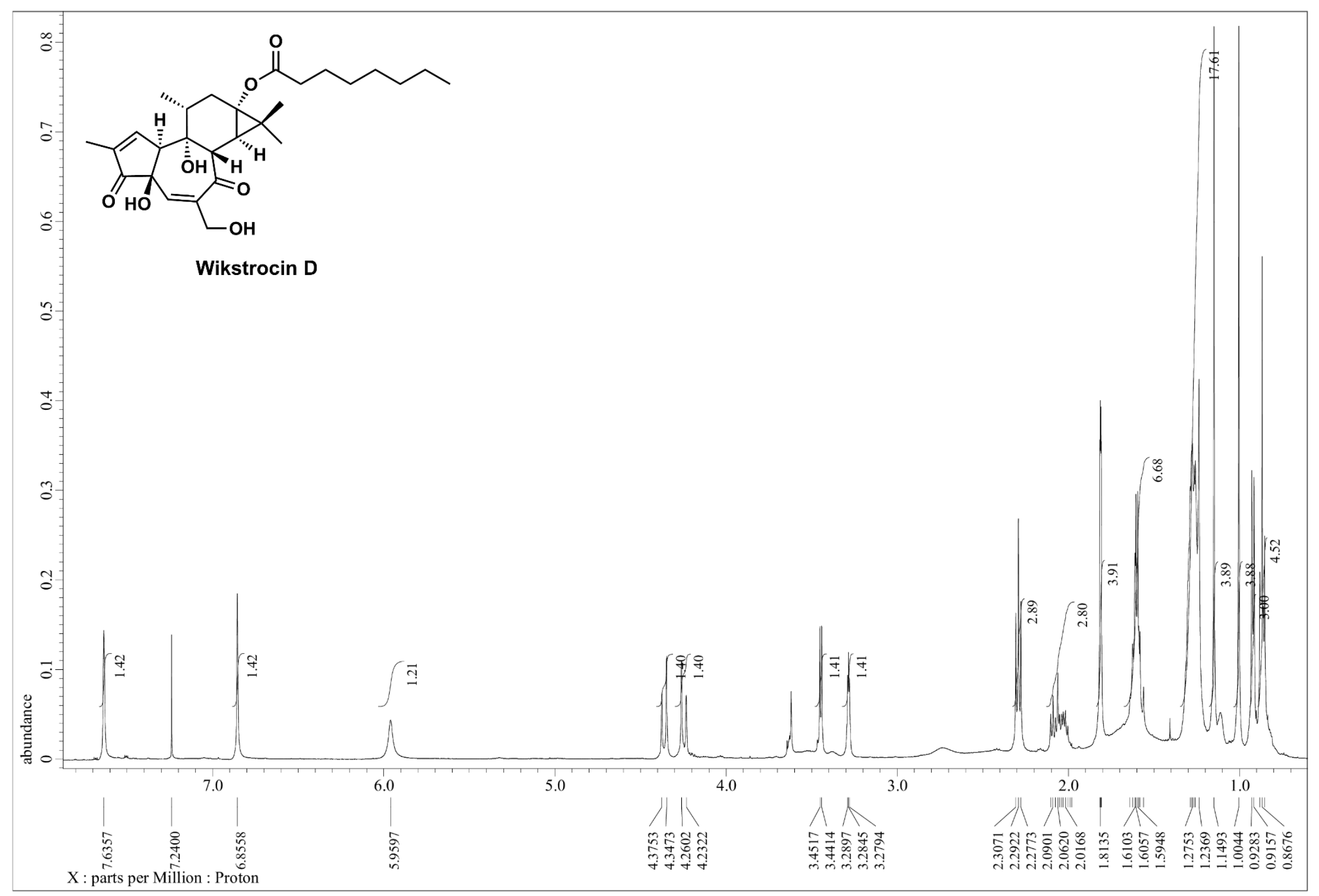

Figure S2. ${ }^{1} \mathrm{H}-\mathrm{NMR}$ Spectrum of compound 1. 


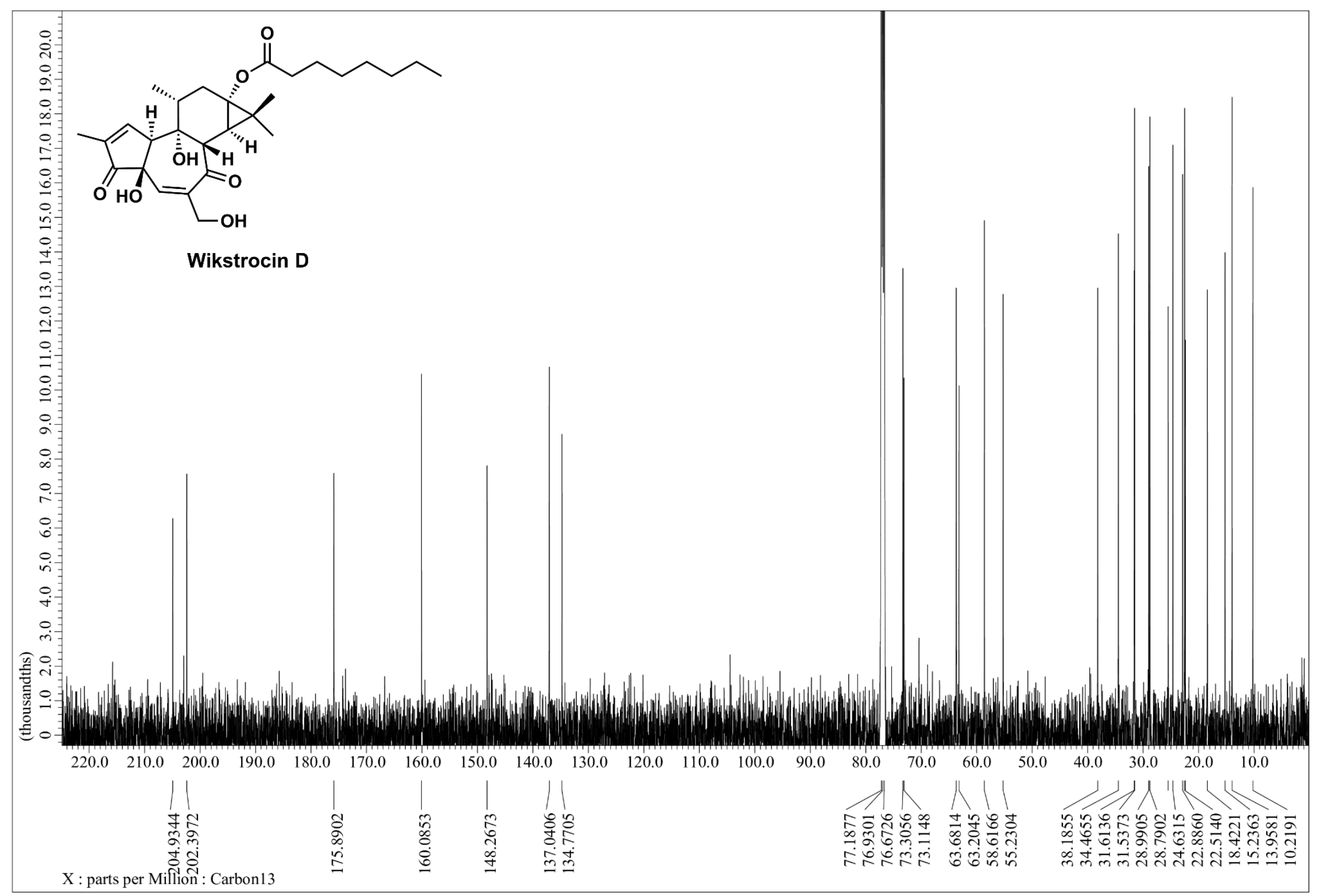

Figure S3. ${ }^{13} \mathrm{C}-\mathrm{NMR}$ Spectrum of compound $\mathbf{1 .}$ 


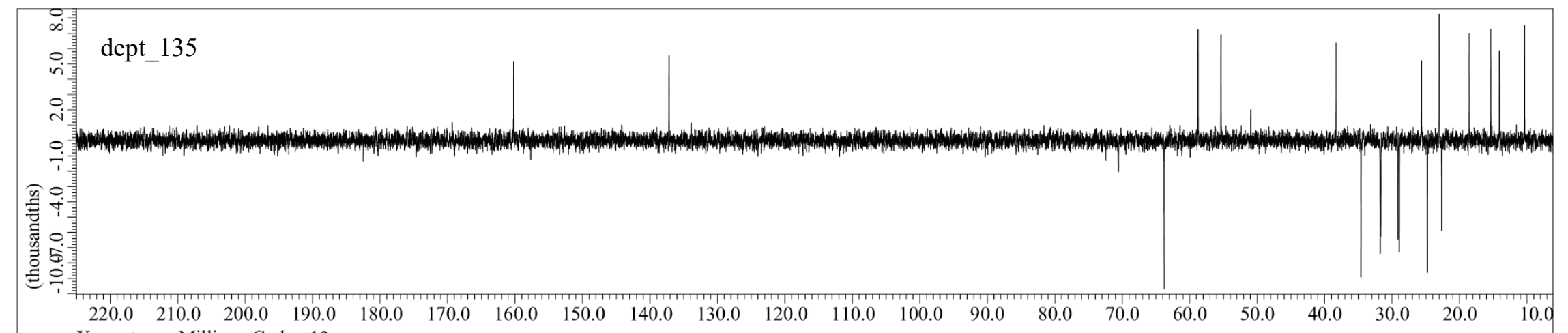

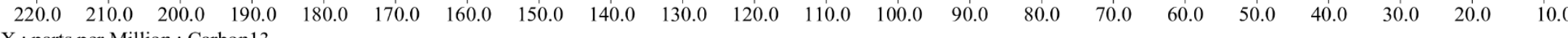

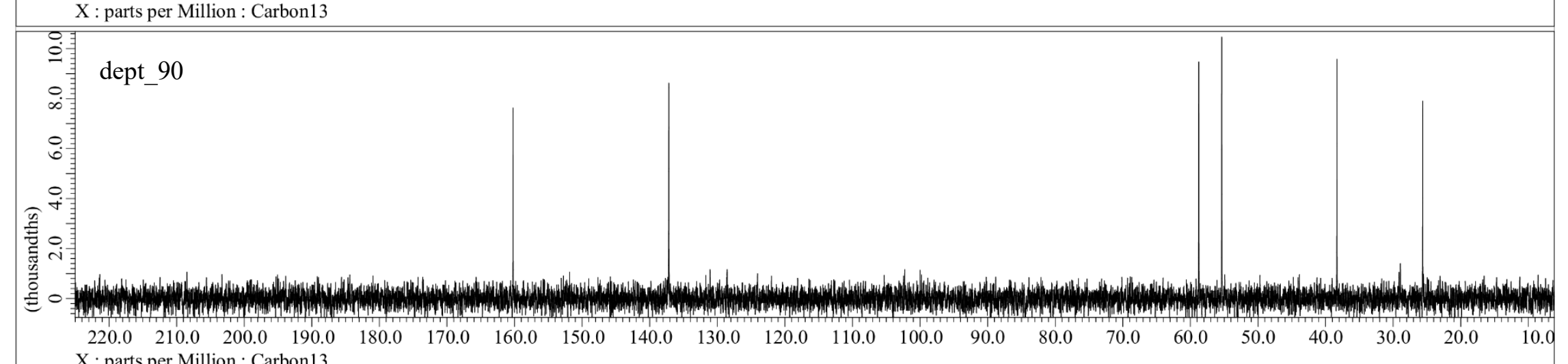

$\mathrm{X}$ : parts per Million : Carbon 13

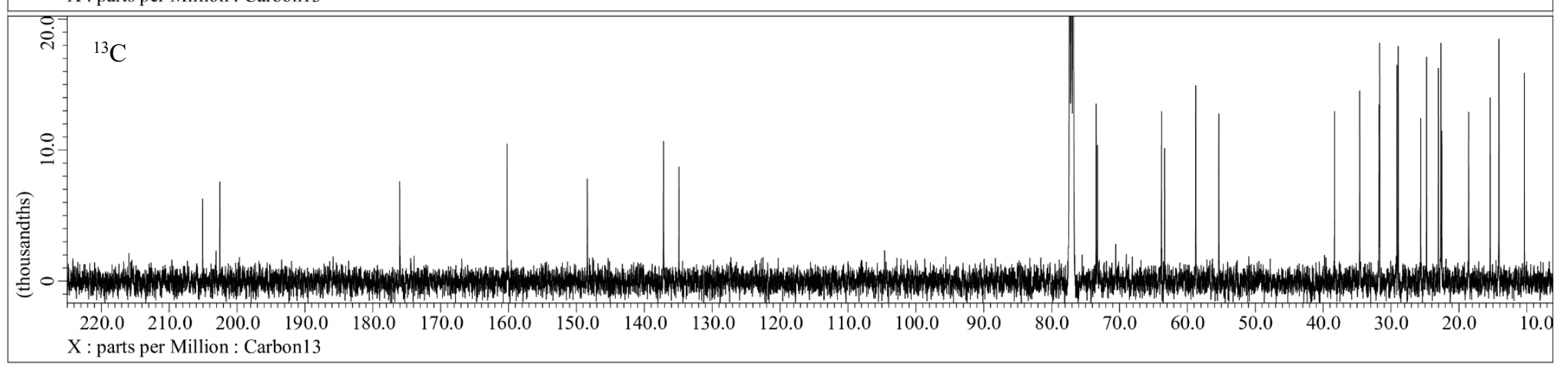

Figure S4. DEPT Spectrum of compound 1. 


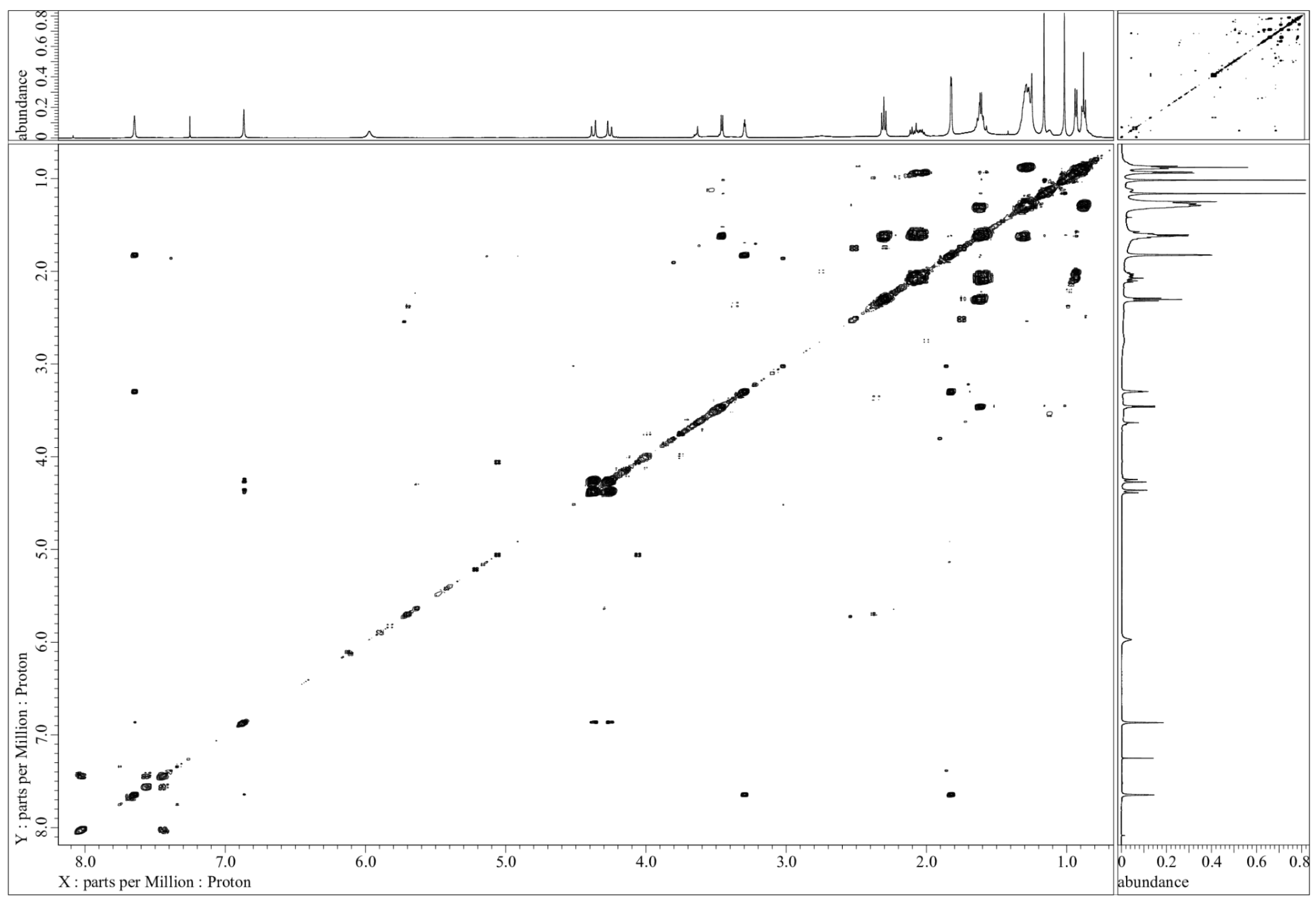

Figure S5. DQF-COSY Spectrum of compound 1.

S10 


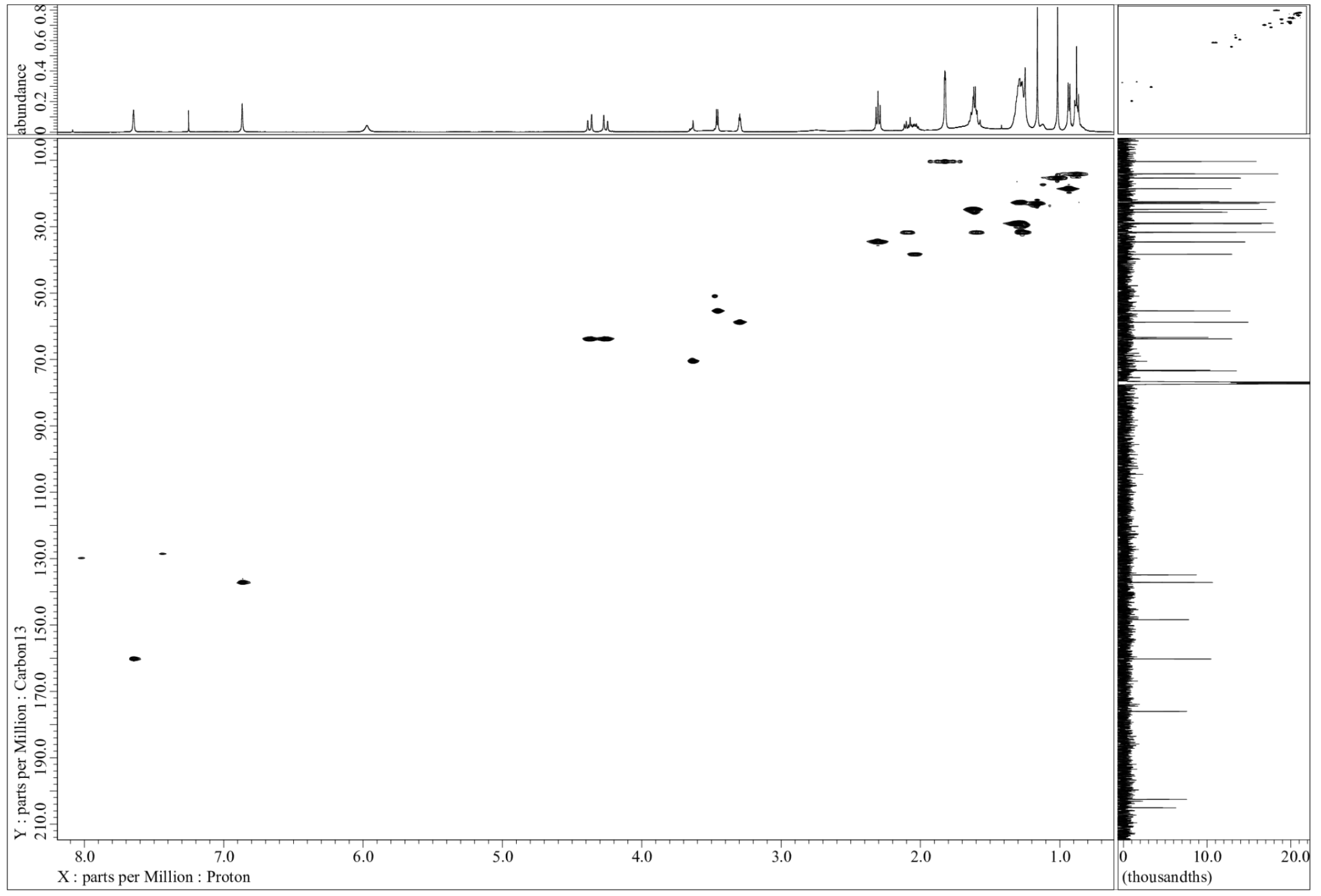

Figure S6. HSQC Spectrum of compound $\mathbf{1 .}$ 


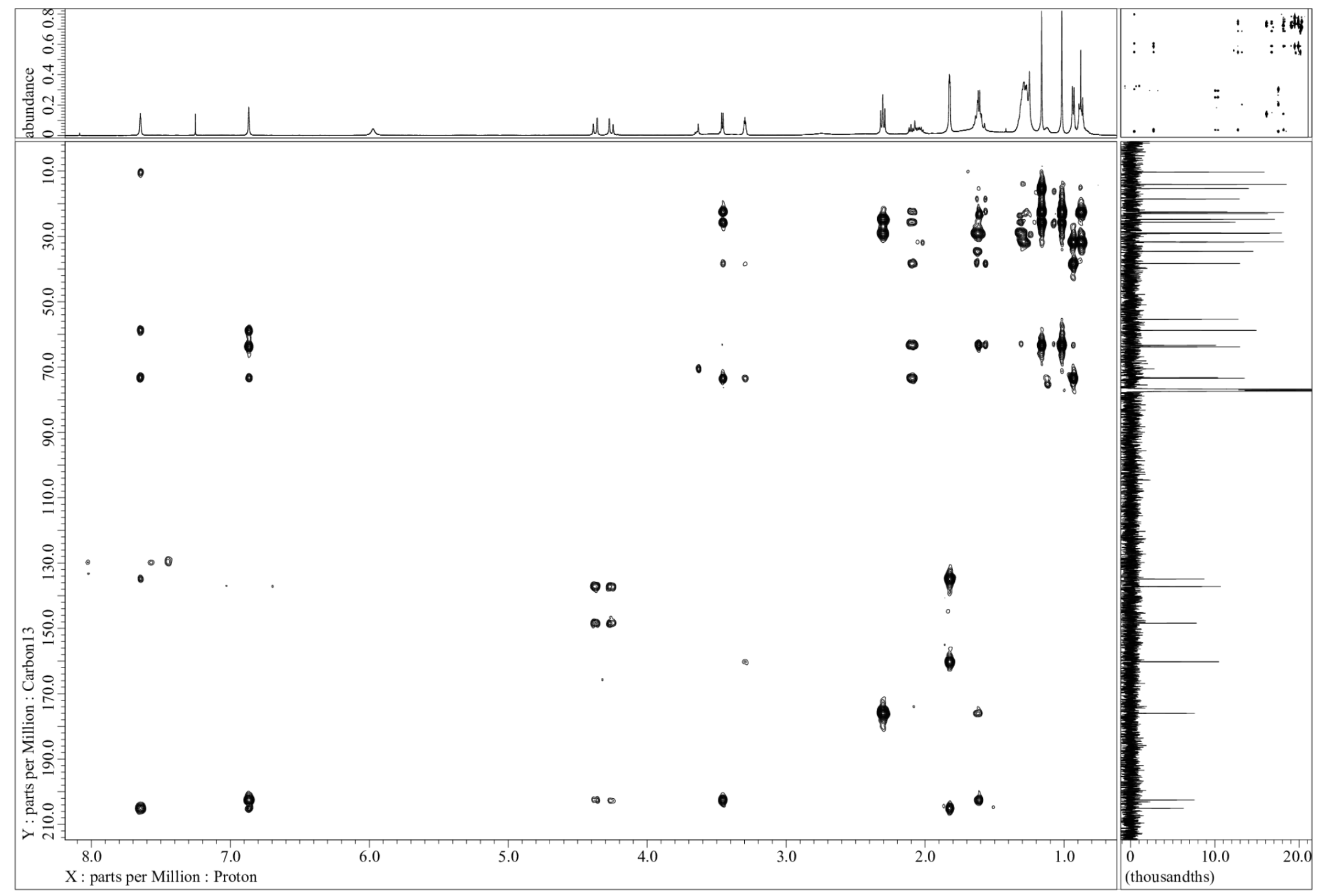

Figure S7. HMBC Spectrum of compound 1. 


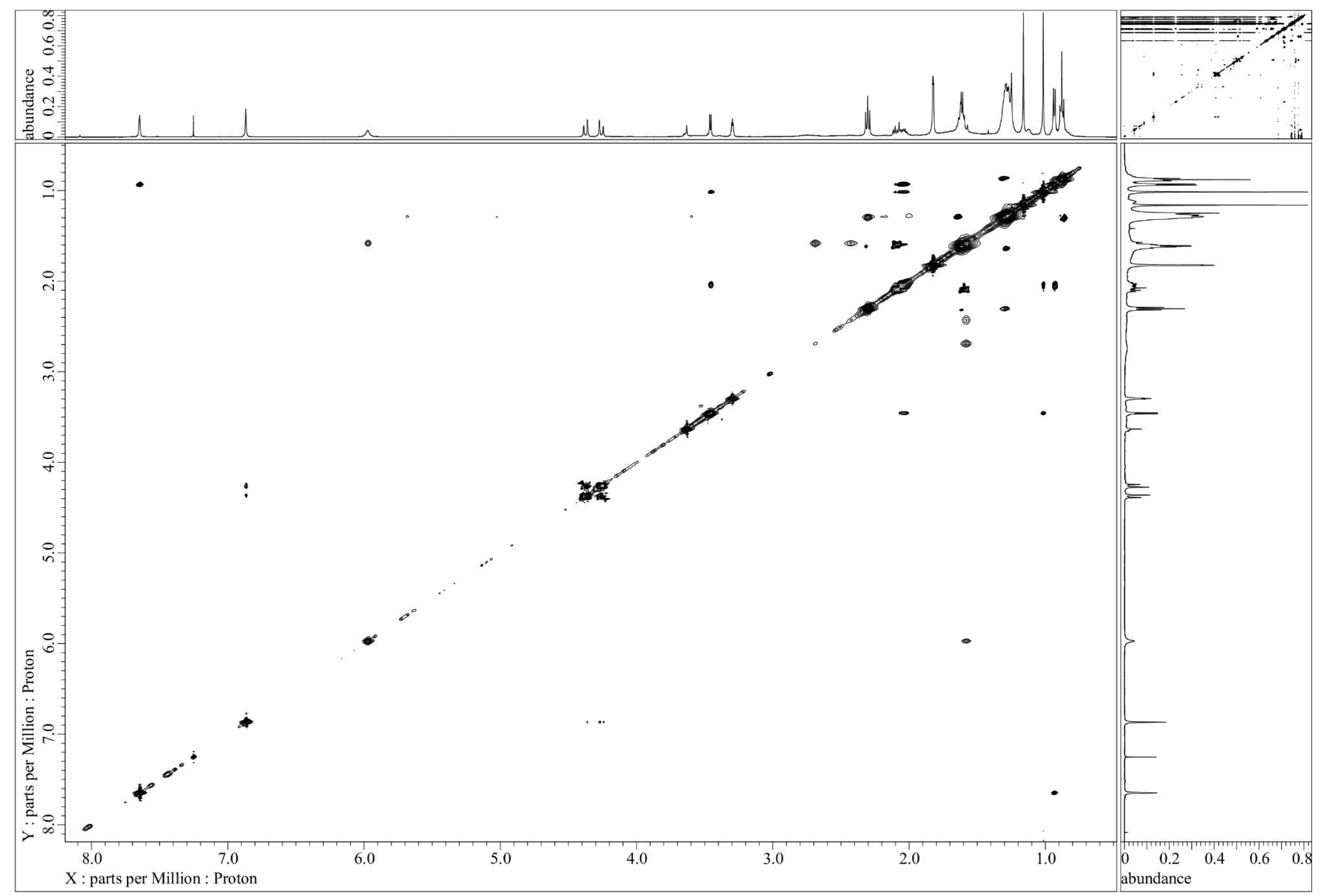

Figure S8. NOESY Spectrum of compound 1. 
$\mathrm{m} / \mathrm{z}=528.2754-538.2754$

Isotope Min Max

$\mathrm{N}-14 \quad 0 \quad 1$

$0-16 \quad 0 \quad 30$

C-12 $0 \quad 50$

H-1 $\quad 0 \quad 100$

Charge -1

Mass tolerance $5.00 \mathrm{ppm}$

Nitrogen rule not used

RDB equiv $-1.00-100.00$

max results 10
$\mathrm{m} / \mathrm{z}$ Theo.
Mass
Delta RDB
(ppm) equiv.
Composition

$533.2754 \quad 533.2756 \quad-0.41 \quad 9.5 \mathrm{C}_{29} \mathrm{H}_{41} \mathrm{O}_{9}$

20200907_WLAd_Full \#779 RT: 7.95 AV: 1 NL: $1.81 \mathrm{E}+007$

T: FTMS + p ESI Full ms [150.0000-2000.0000]

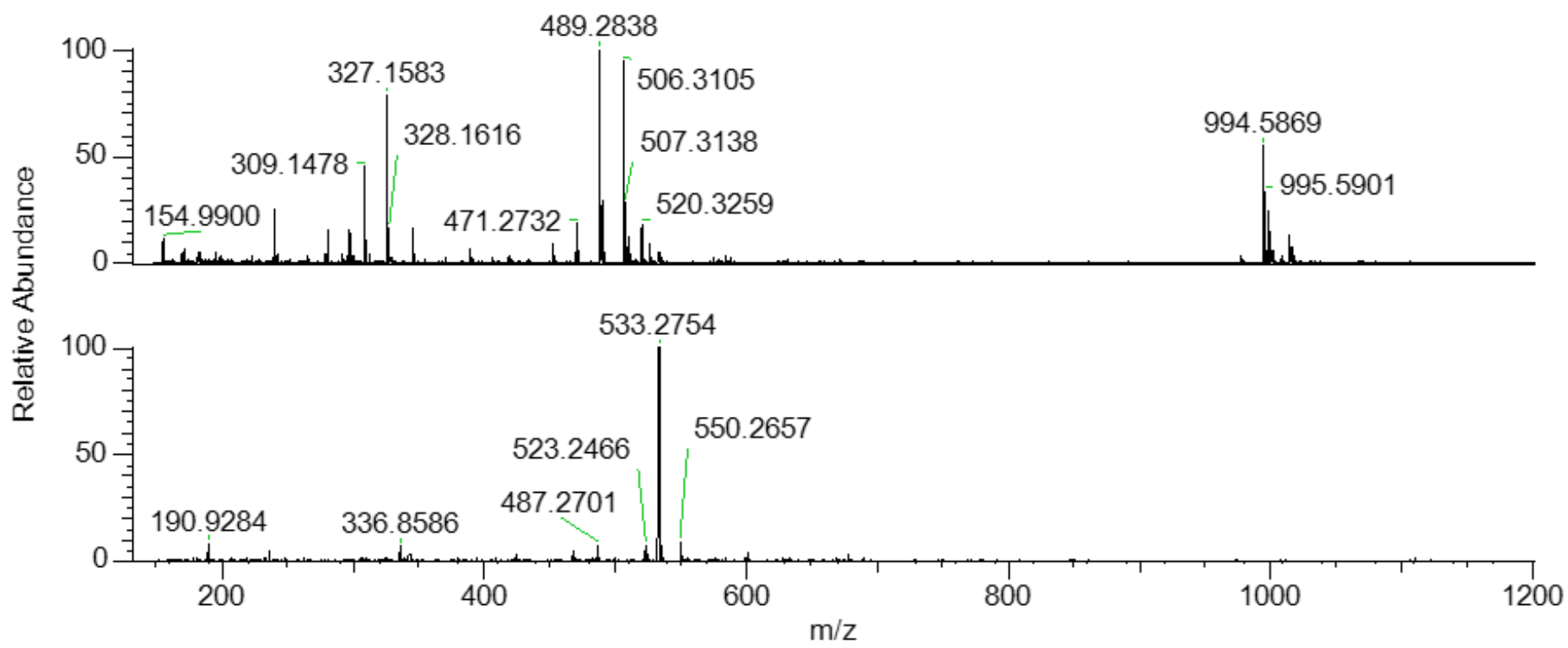

$\mathrm{NL}: 1.81 \mathrm{E} 7$

20200907_WLAd_Full \#779 RT: 7.95 AV: 1

T: FTMS + p ESI Full ms

[150.0000-2000.0000]

NL: $2.17 \mathrm{E} 7$

20200907_WLAd_Full \#780

RT: 7.96 AV: 1

T: FTMS - p ESI Full ms

150.0000-2000.0000]

Figure S9. HRESIMS Spectrum of compound 1. 


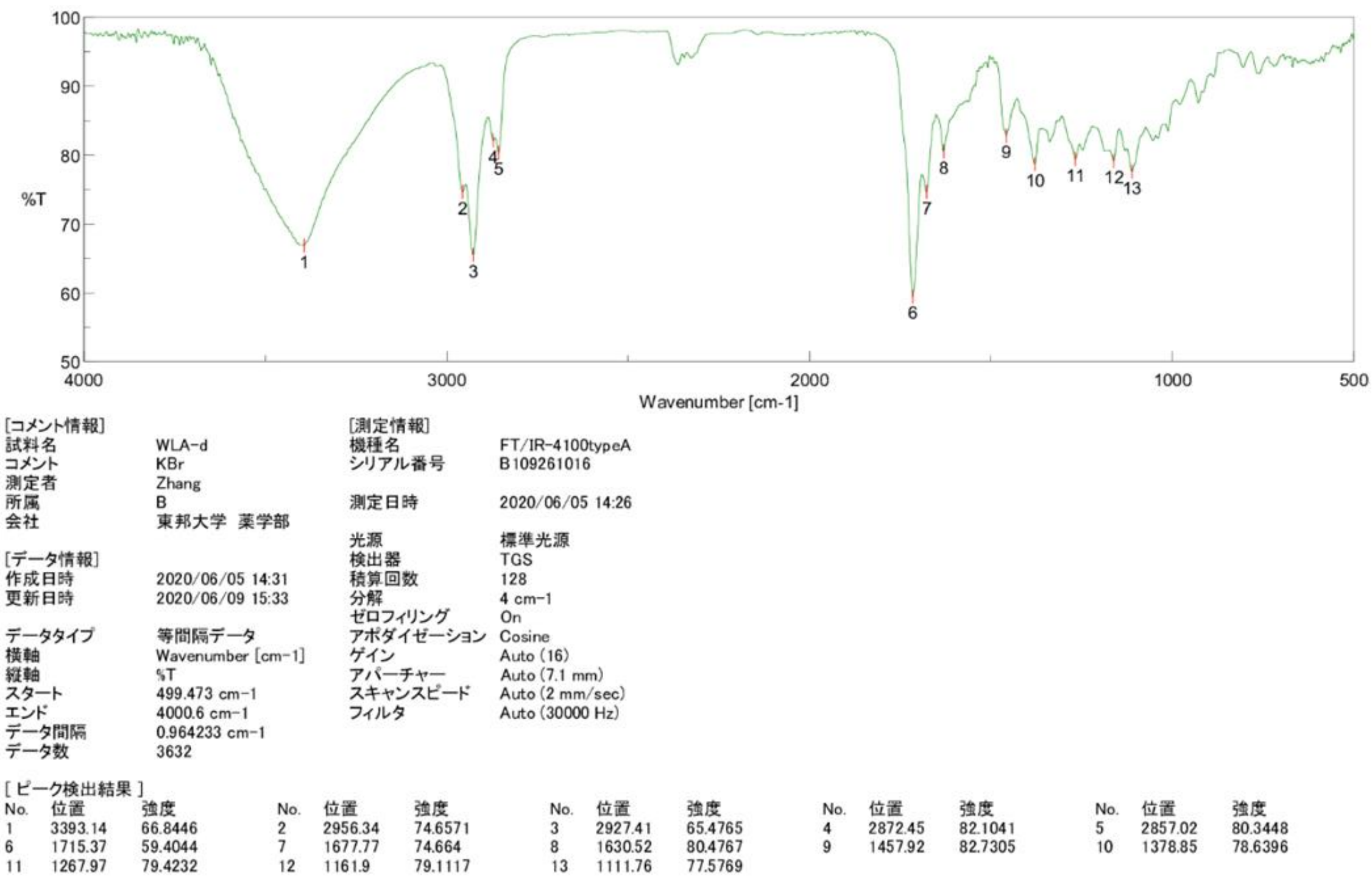

Figure S10. IR Spectrum of compound 1. 


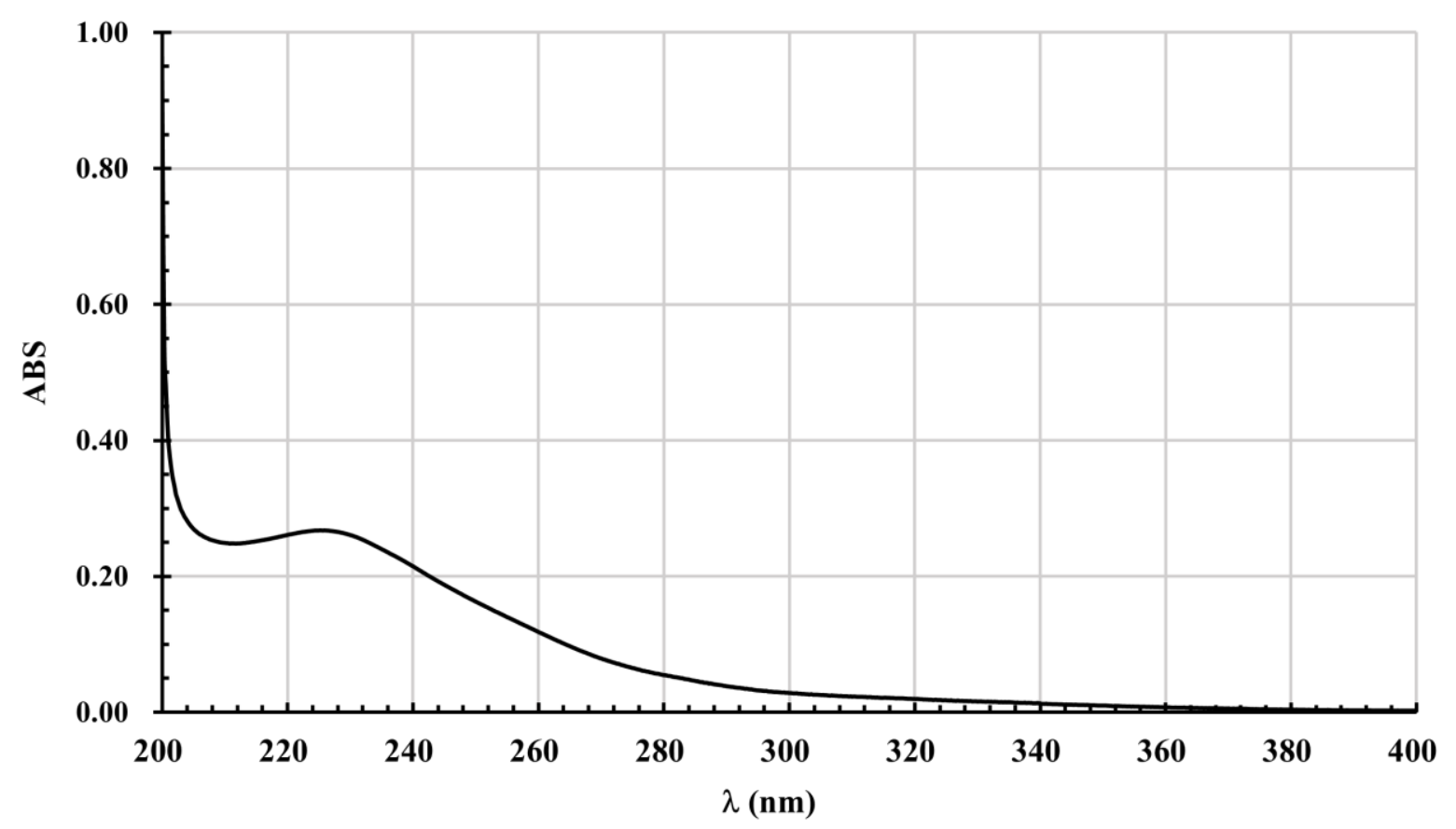

Figure S11. UV Spectrum of compound 1.

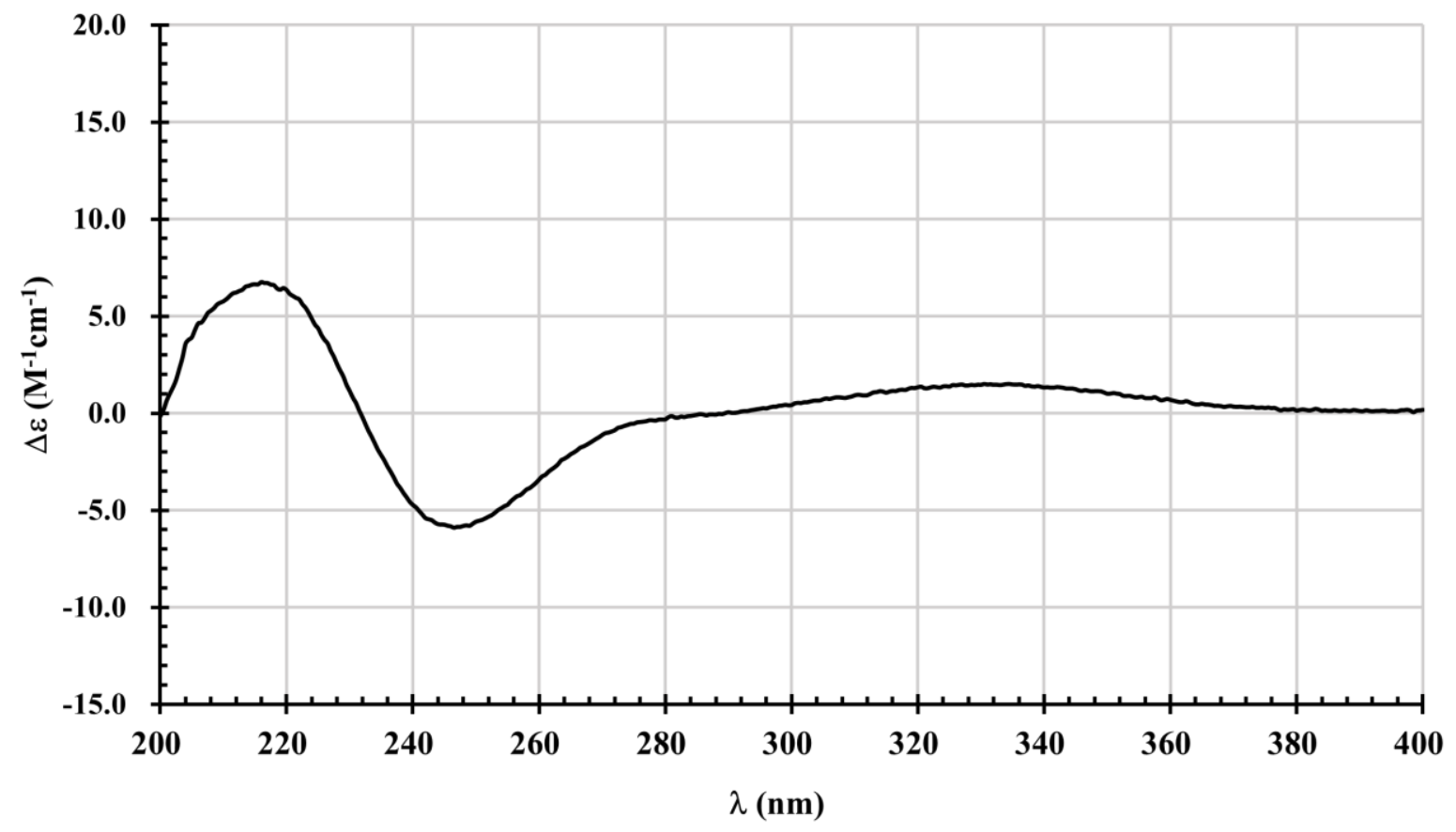

Figure S12. ECD Spectrum of compound 1. 


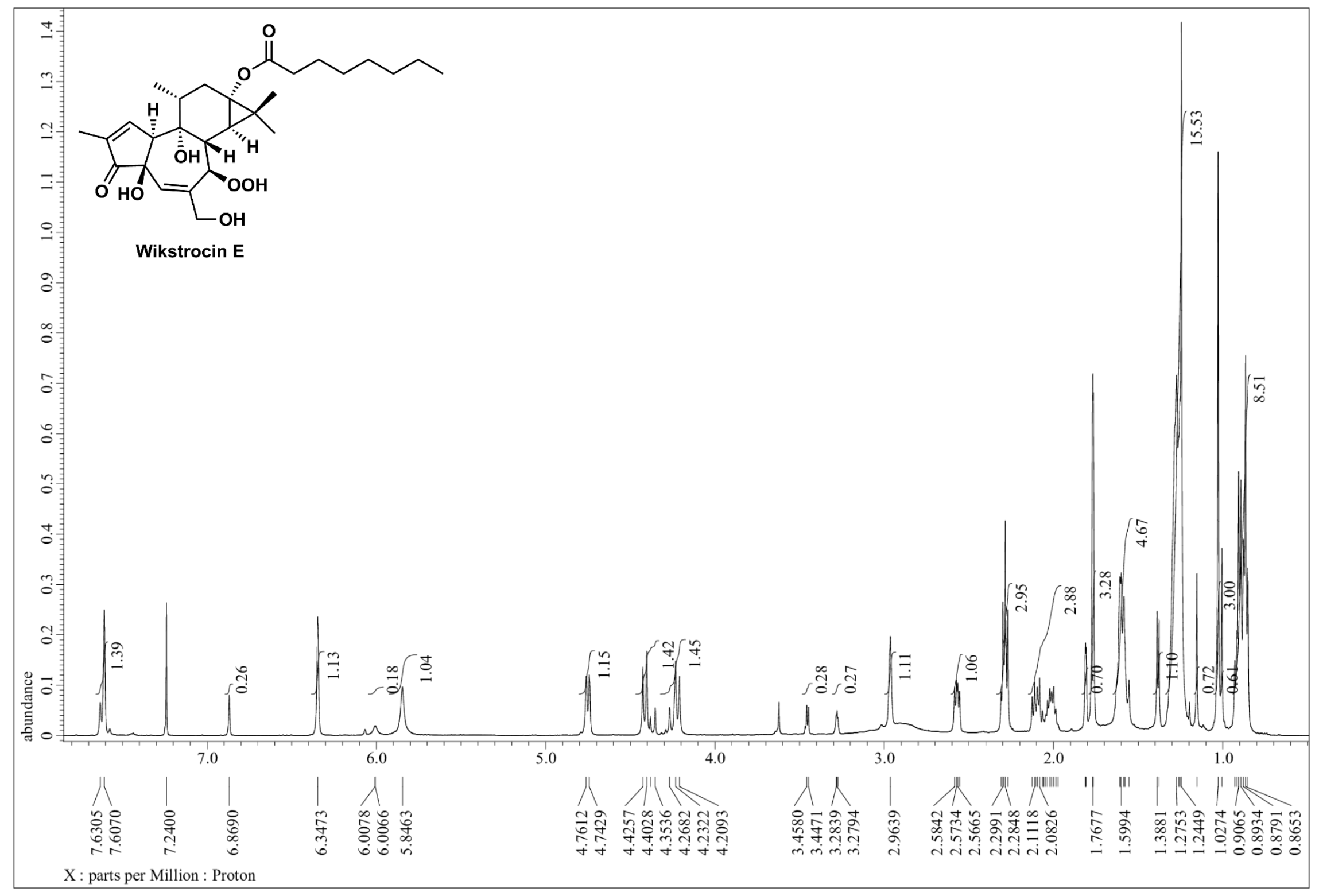

Figure S13. ${ }^{1} \mathrm{H}-\mathrm{NMR}$ Spectrum of compound 2. 


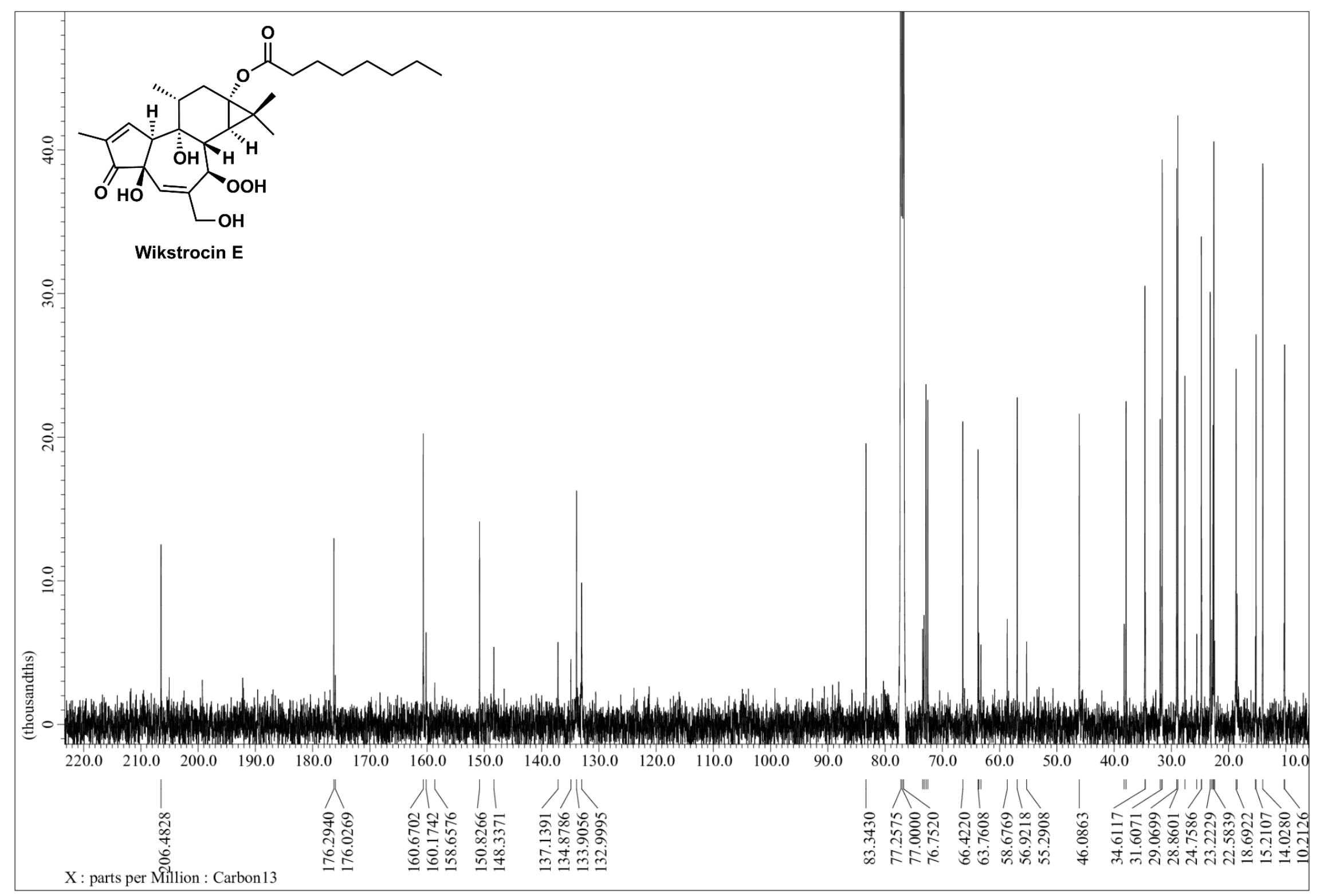

Figure S14. ${ }^{13} \mathrm{C}-\mathrm{NMR}$ Spectrum of compound 2. 


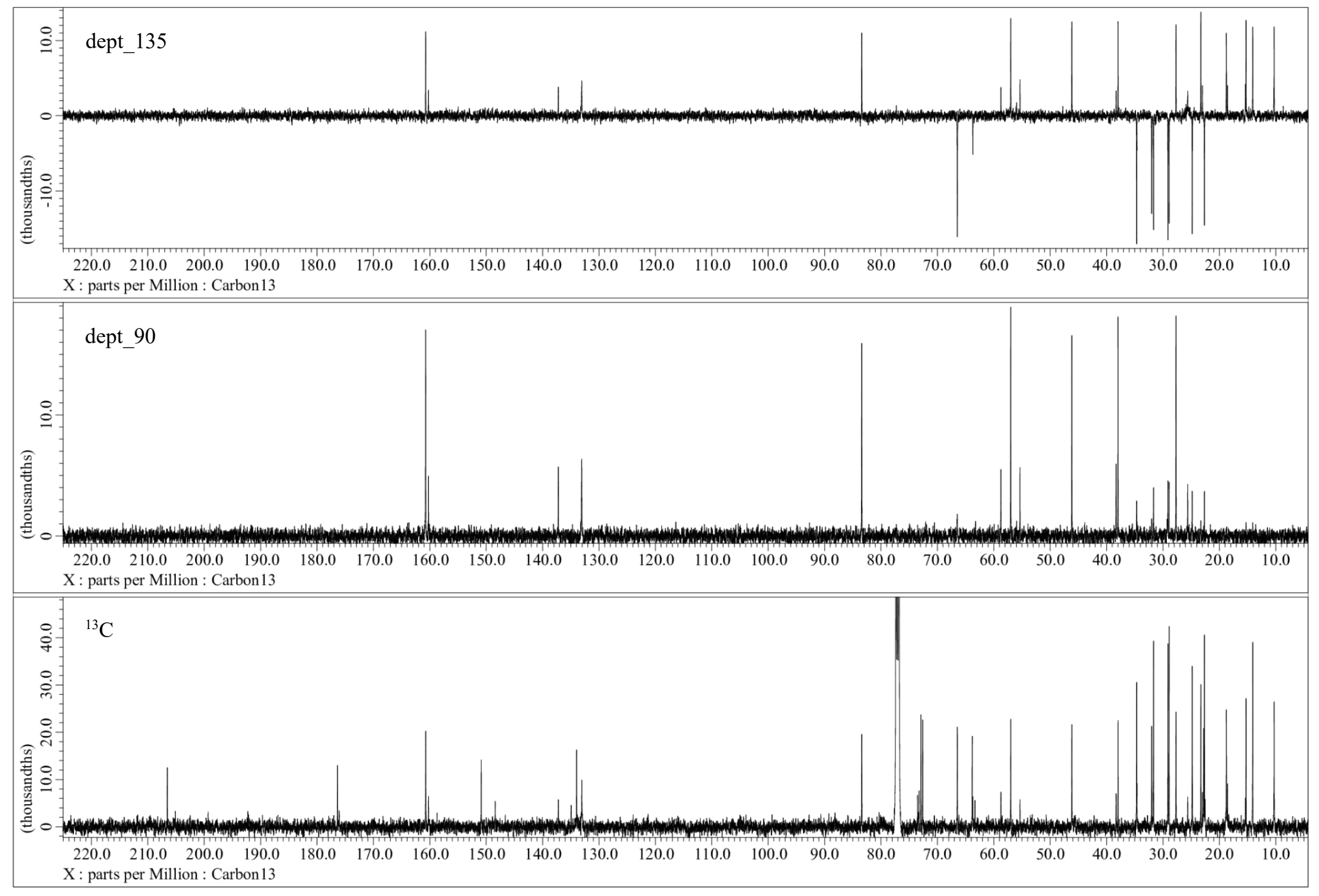

Figure S15. DEPT Spectrum of compound 2. 


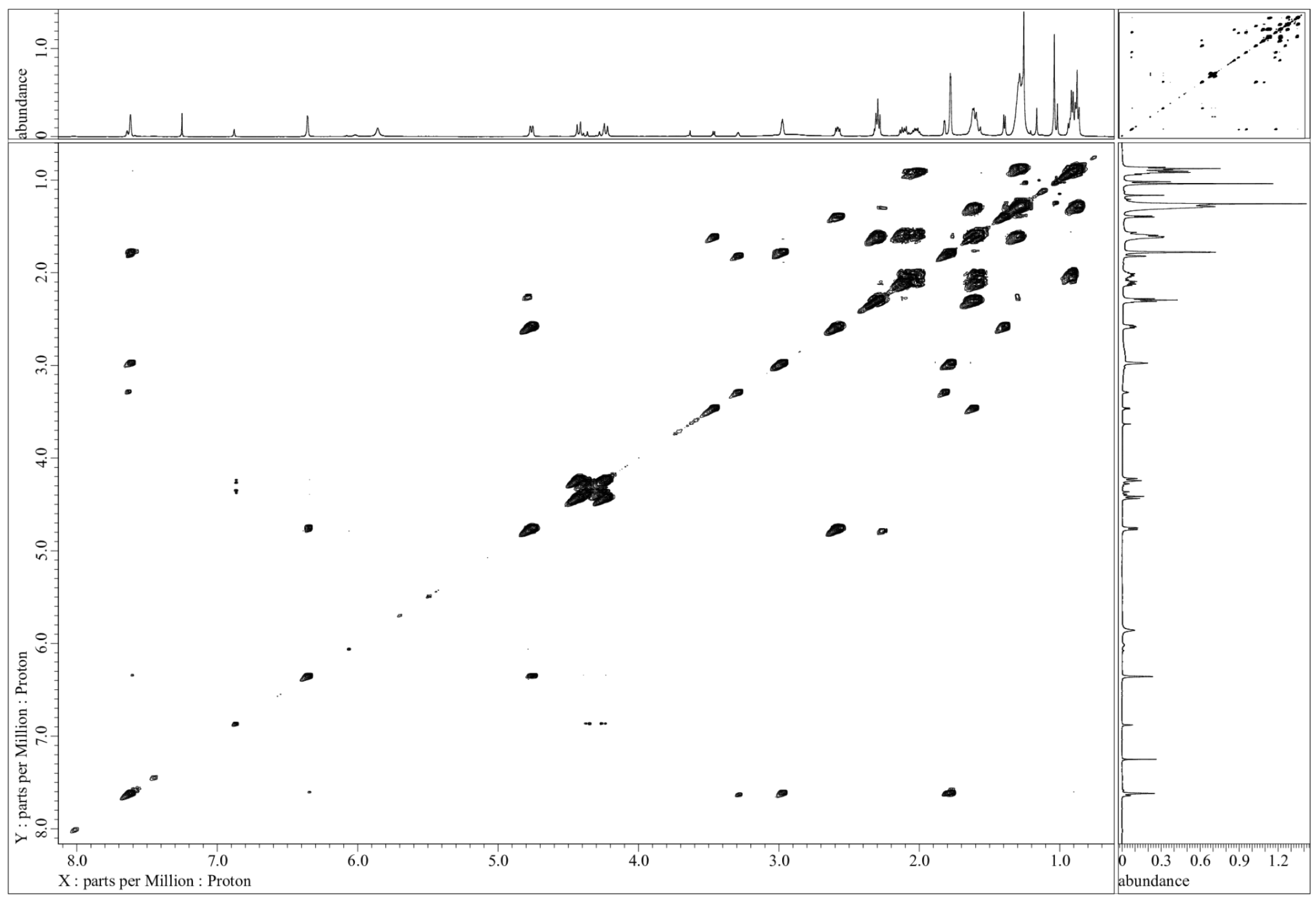

Figure S16. DQF-COSY Spectrum of compound 2.

S20 


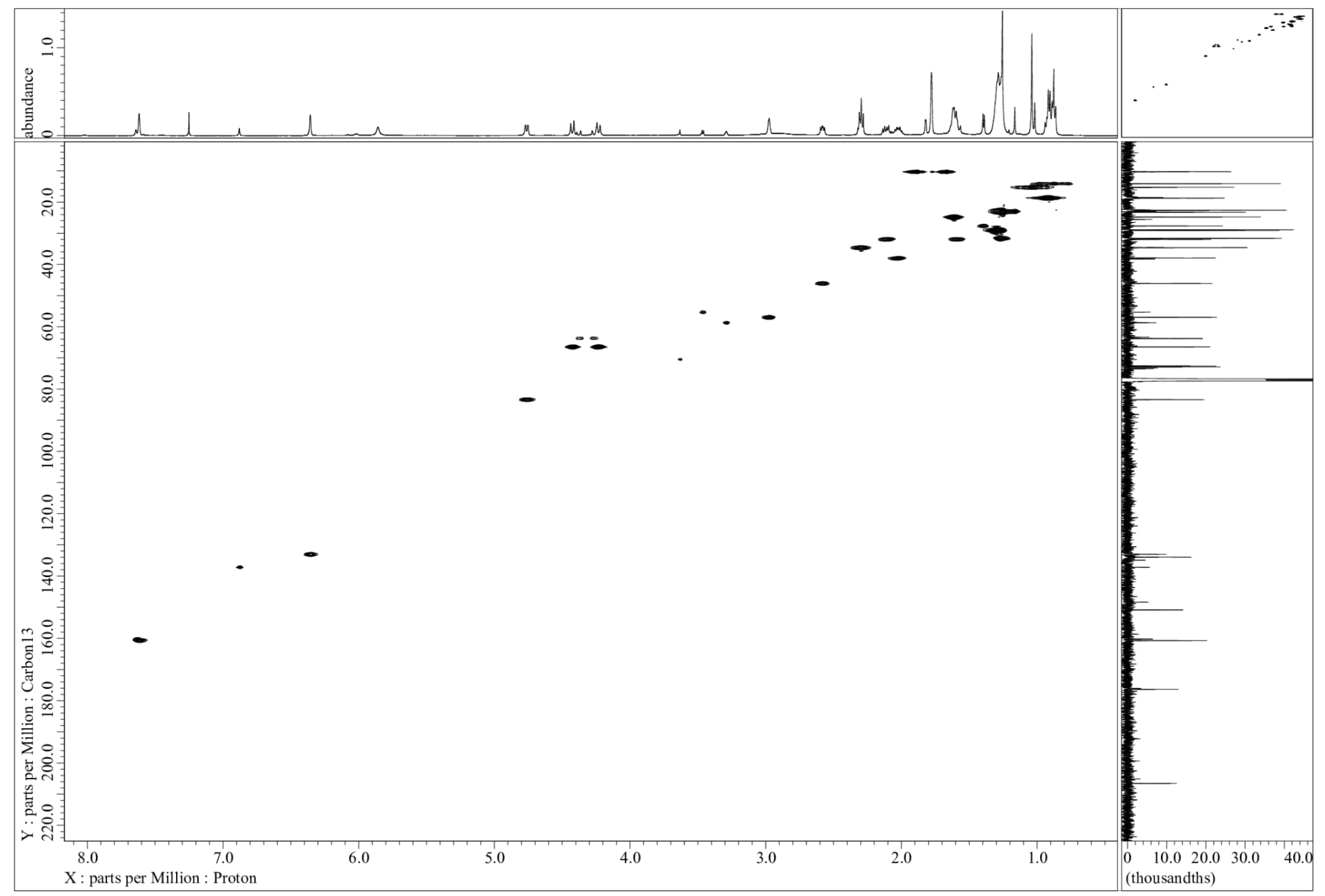

Figure S17. HSQC Spectrum of compound 2. 


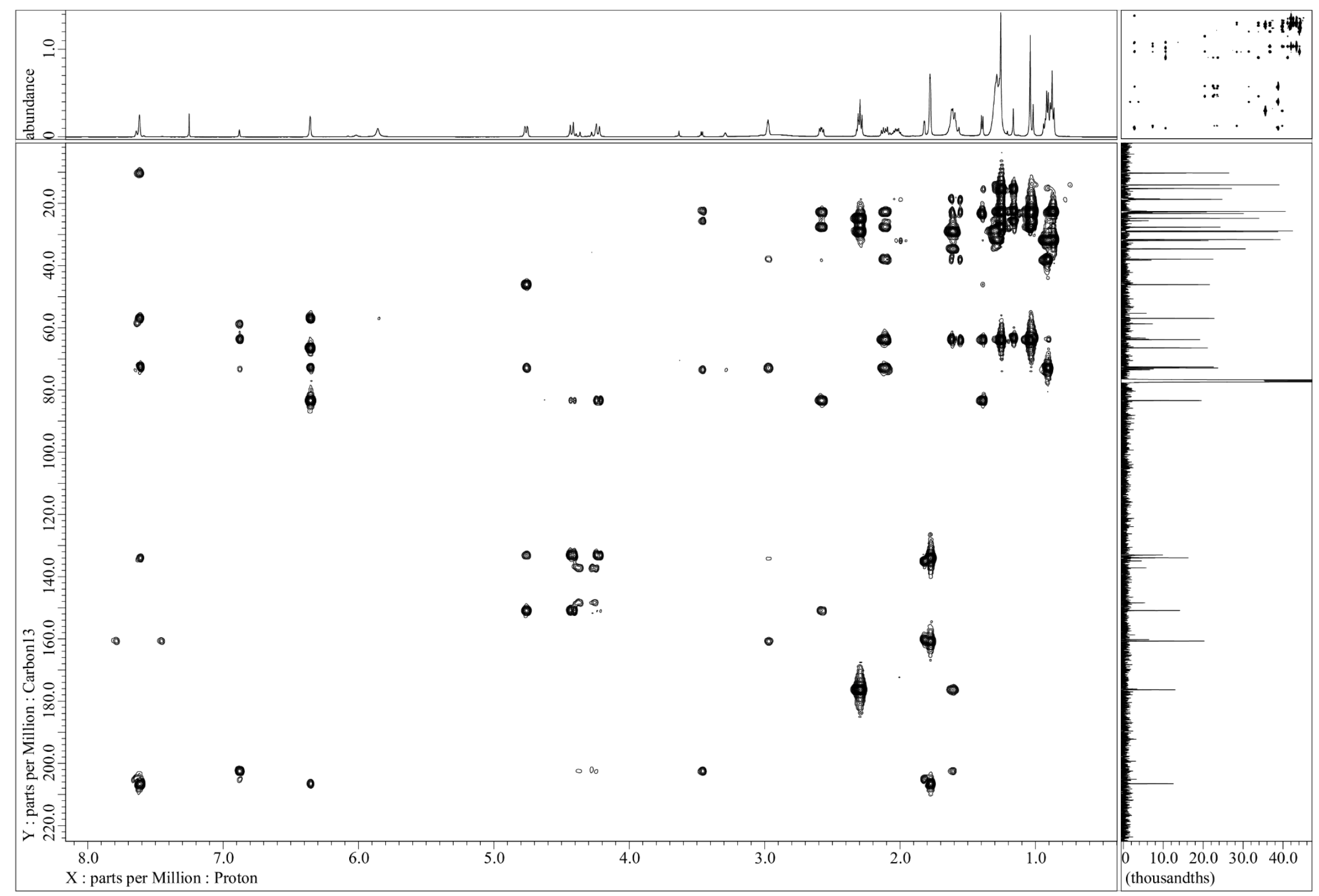

Figure S18. HMBC Spectrum of compound 2. 


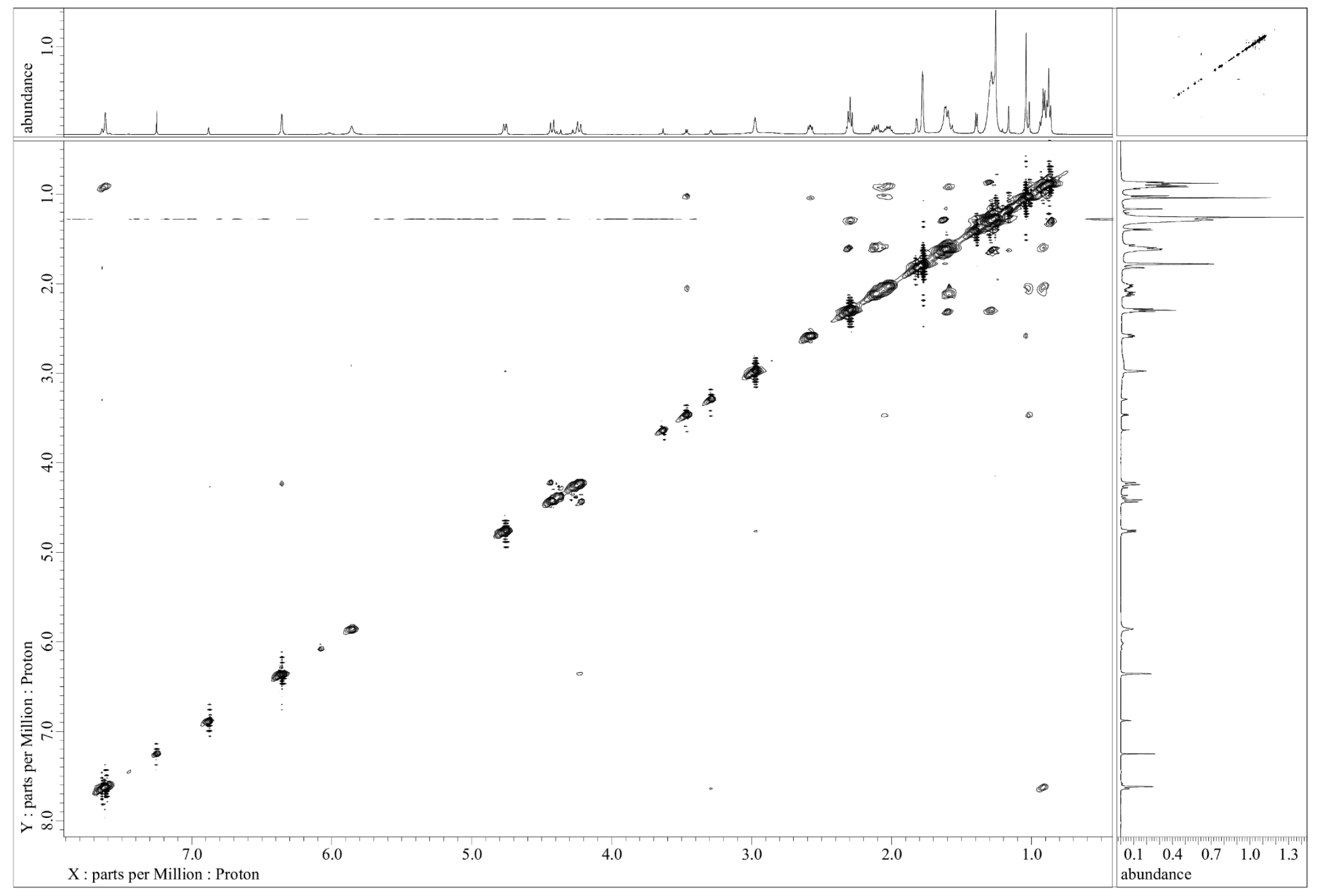

Figure S19. NOESY Spectrum of compound 2. 


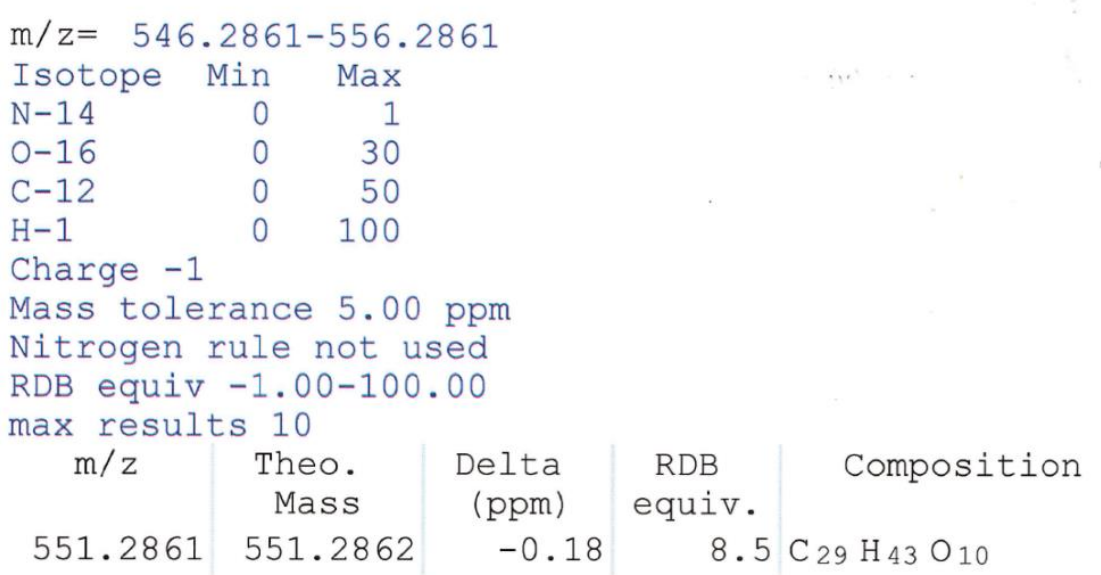

20200907_WLAn_Full \#765 RT: 7.81 AV: 1 NL: 1.32E+007

T: FTMS + p ESI Full ms [150.0000-2000.0000]

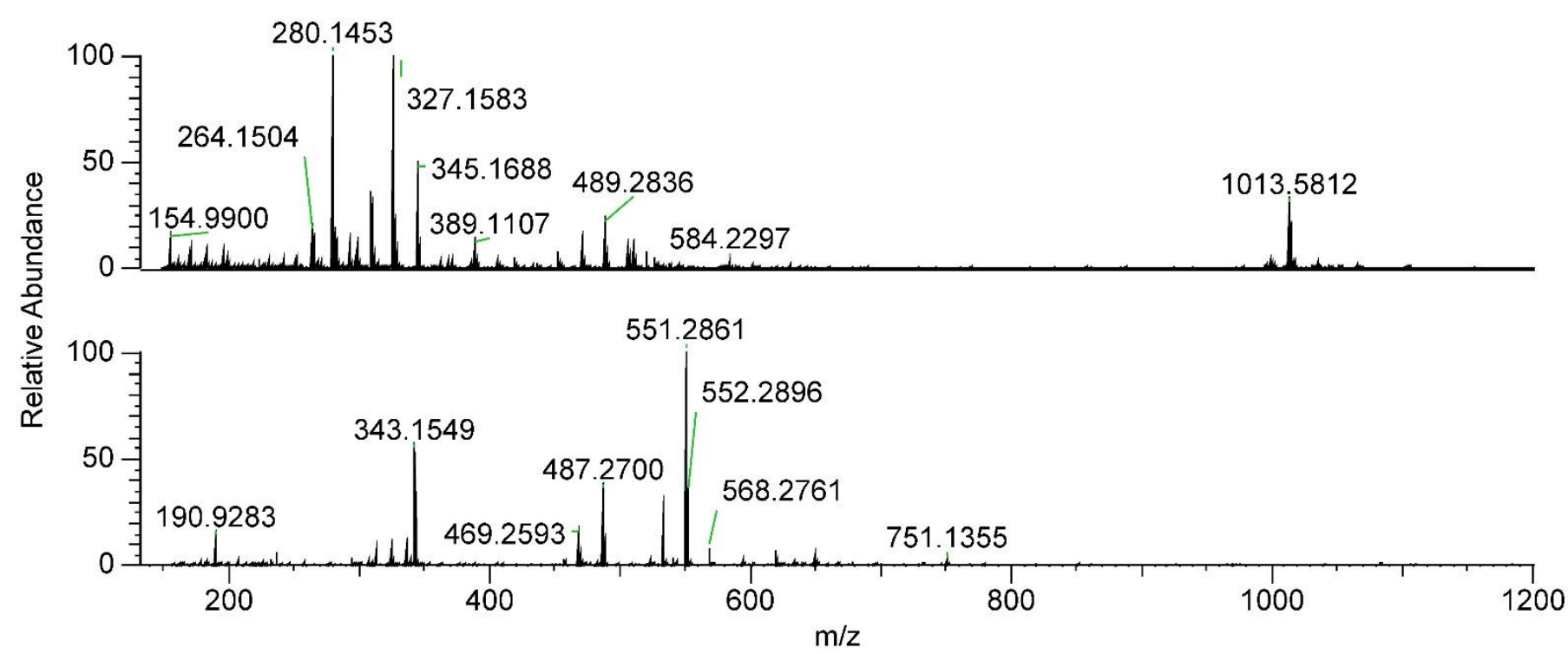

NL: $1.32 \mathrm{E} 7$

20200907_WLAn_Full \#765

RT: 7.81 AV: 1

T: FTMS + p ESI Full ms

[150.0000-2000.0000]

$\mathrm{NL}: 1.33 \mathrm{E} 7$

20200907 WLAn Full \#766 RT: 7.82 AV: 1

T: FTMS - p ESI Full ms

[150.0000-2000.0000]

Figure S20. HRESIMS data of compound 2. 


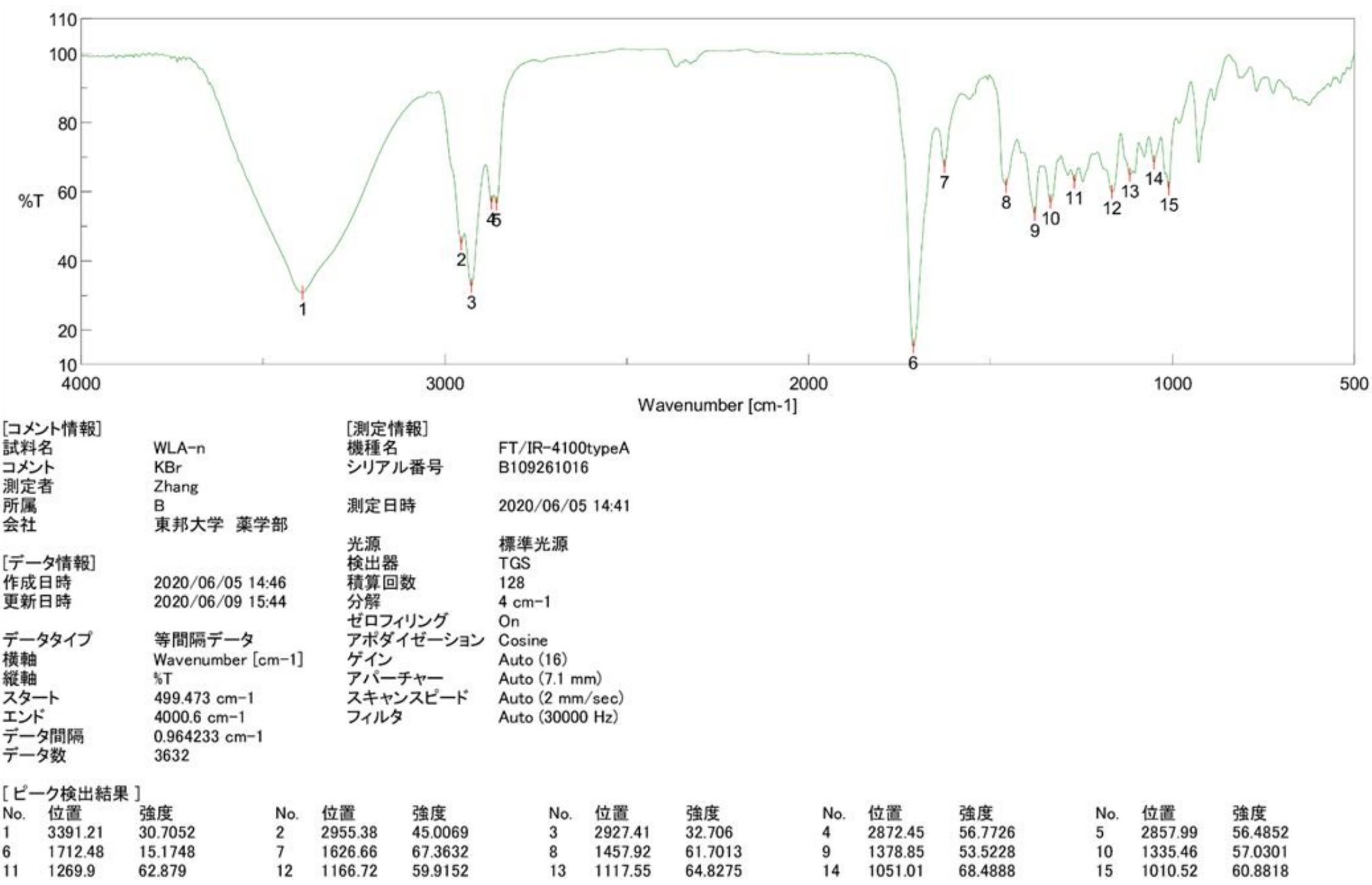

Figure S21. IR Spectrum of compound 2. 


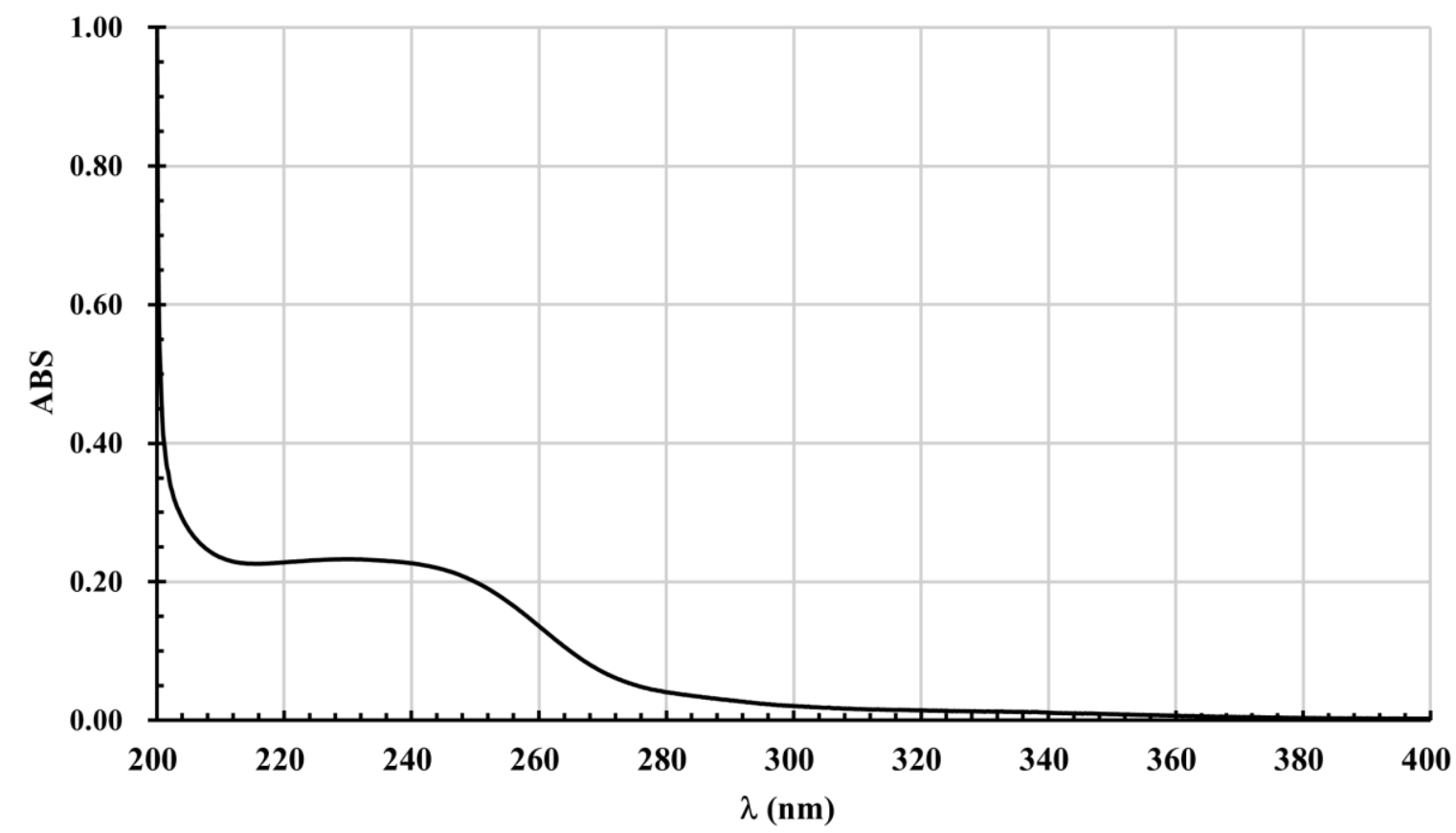

Figure S22. UV Spectrum of compound 2.

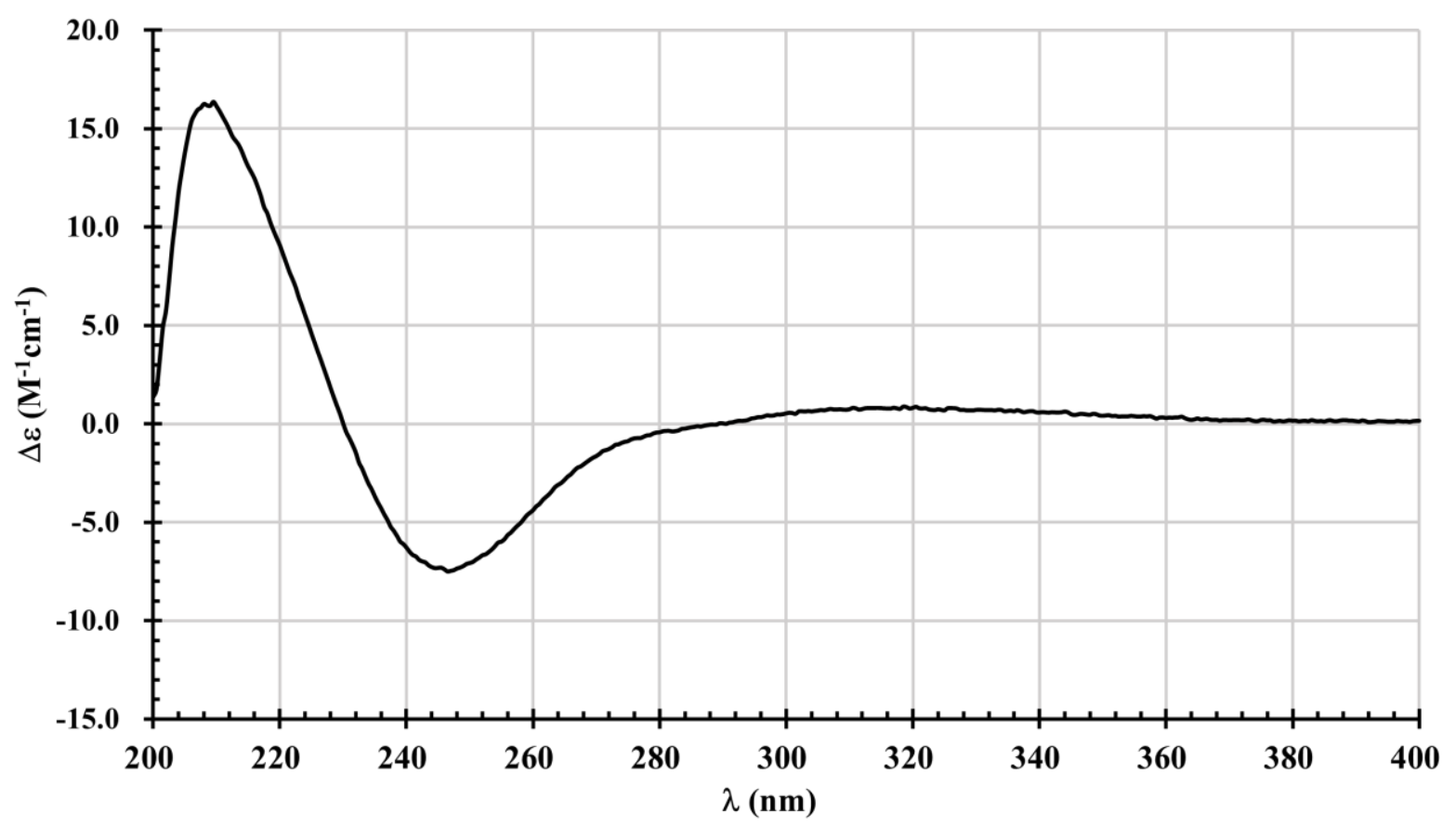

Figure S23. ECD Spectrum of compound 2. 